\title{
Horospherical model for holomorphic discrete series and horospherical Cauchy transform
}

\author{
Simon Gindikin, Bernhard Krötz and Gestur Ólafsson
}

\begin{abstract}
We define a complex horospherical transform on an affine symmetric space $X=G / H$ of Hermitian type and show that it has no kernel on the representations of the $H$-spherical holomorphic discrete series.
\end{abstract}

\section{Introduction}

For some homogeneous spaces the method of horospheres delivers an effective way to decompose representations into irreducible representations. For Riemannian symmetric spaces $Y=G / K$, horospheres are orbits of maximal unipotent subgroups of $G$. They are parameterized by points of the horospherical homogeneous space $\Xi_{\mathbb{R}}=G / M N$ where $N$ is a fixed maximal unipotent subgroup and $M=Z_{K}(A)$ as usual. The horospherical transform maps sufficiently regular functions on $Y$ to the corresponding average along the horospheres. The crucial point is that the abelian group $A$ acts on $\Xi$ and that this action commutes with the action of $G$. The decomposition of the natural representation of $G$ in $L^{2}(\Xi)$ into irreducible representations reduces to the decomposition relative to $A$. In this way we obtain all unitary spherical representations on $Y$ (with constant multiplicity), except the complementary series. The computation of the Plancherel measure on $Y$ is equivalent to the inversion of the horospherical transform.

The method of horospheres works for several other types of homogeneous spaces, including complex semisimple Lie groups (considered as symmetric spaces) but it has very serious restrictions: discrete series representations lie in the kernel of the horospherical transform, as well as all representations induced from parabolic subgroups that are not minimal. In short, the kernel is the orthocomplement of the most continuous part of the spectrum. The simplest example when the horospherical transform can not be inverted is for the group $\mathrm{SL}(2, \mathbb{R})$. In [Gin00, Gin02, Gin04], a modification of the method of horospheres was suggested: the complex horospherical transform (the horospherical Cauchy-Radon transform). For a homogeneous space $X$ we consider the complexification $X_{\mathbb{C}}$ and, instead of real horospheres on $X$, we consider complex horospheres on $X_{\mathbb{C}}$ without real points (they do not intersect $X$ ). The integration along a real horosphere may be viewed as the integration of a $\delta$-function on $X$ with support on this horosphere. In the complex version, we replace this $\delta$-function by a Cauchy-type kernel with singularities on the complex horosphere without real points. In [Gin00, Gin02] it was shown that such a complex horospherical transform is injective for $\mathrm{SL}(2 ; \mathbb{R})$ and that it reproduces the Plancherel formula; in [Gin04] it was shown for all compact symmetric spaces.

Received 7 December 2004, accepted in final form 30 September 2005.

2000 Mathematics Subject Classification 22E46.

Keywords: semisimple Lie groups, symmetric spaces, horospheres, horospherical transform, Cauchy kernel, Hardy spaces.

The first author was supported by the Louisiana Board of Regents grant Visiting Experts in Mathematics. The second and third authors were supported by the Research in Pairs program of the Mathematisches Forschungsinstitut, Oberwolfach. The third author was also supported by NSF grant DMS-0139783 and DMS-0402068.

This journal is (c) Foundation Compositio Mathematica 2006. 


\section{S. Gindikin, B. Krötz And G. Ólafsson}

The objective of this paper is to show that the complex horospherical transform has no kernel on the holomorphic discrete series. Holomorphic discrete series exist for affine symmetric spaces $X=G / H$ of Hermitian type. Here $G$ is a group of Hermitian type [Har56, OO91]. The corresponding part of $L^{2}(X)$ can be realized as boundary values of the Hardy space $\mathcal{H}^{2}\left(D_{+}\right)$in a Stein tube $D_{+} \subset X_{\mathbb{C}}$ with edge $X$ (see [HOO91]). Our aim is to define a complex horospherical transform that has no kernel on the representations of the $H$-spherical holomorphic discrete series.

The first step towards the realization of our objective is the construction of the space that is going to be the image of the complex horospherical transform. For this, we consider those complex horospheres in the Stein symmetric space $X_{\mathbb{C}}=G_{\mathbb{C}} / H_{\mathbb{C}}$ that are parameterized by points of the complex horospherical space $\Xi=G_{\mathbb{C}} / M_{\mathbb{C}} N_{\mathbb{C}}$. In $\Xi$, we then consider an orbit $\Xi_{+}$of $G \times \mathcal{T}_{+}$where $\mathcal{T}_{+}$is a semigroup in the complex abelian group $T_{\mathbb{C}}=A T$ with the compact torus $T$ as the edge. The space $\mathcal{O}\left(\Xi_{+}\right)$of holomorphic functions on $\Xi_{+}$is a Fréchet model of the holomorphic discrete series. More exactly, if we decompose this representation with respect to the compact torus $T$, we obtain $G$-modules that are lowest weight modules (if they are irreducible); we obtain all such modules with multiplicity one. Using the abelian semigroup $\mathcal{T}_{+}$we can define a Hardy-type space $\mathcal{H}^{2}\left(\Xi_{+}\right)$with spectrum 'almost all' of the holomorphic discrete series.

The next step consists of providing the geometrical background for the construction of the horospherical transform. First, we prove that the horospheres $E(\xi)$ parameterized by points $\xi \in \Xi_{+}$ do not intersect $X$. We construct a simple Cauchy-type kernel that has no singularities on $X$ and the edge of its singularities coincides with $E(\xi)$. Using this kernel, we define the horospherical Cauchy transform from $L^{1}(X)$ to $\mathcal{O}\left(\Xi_{+}\right)$, which can be extended on $L^{2}(X)$. The horospherical transform decomposed under $T$ yields the holomorphic spherical Fourier transform.

The last step is the inversion of the horospherical Cauchy transform. We give the Radon-type inversion formula using results from [Kro01] for the holomorphic discrete series. Let us remark that for $X=\mathrm{SL}(2, \mathbb{R})$, the inversion formula was obtained in [Gin00, Gin02] with tools from integral geometry on quadrics. This method automatically extends on any symmetric spaces of Hermitian type of rank 1 , that is, the hyperboloids of signature $(2, n)$. Let us also pay attention to the similarity of formulas in this paper and formulas in [Gin04] for compact symmetric spaces. This confirms the view that finite-dimensional spherical representations are similar to representations of holomorphic discrete series.

\section{Symmetric spaces of Hermitian type}

The objective of this section is to set up a standard choice of terminology that will be used throughout the text.

Let us fix some conventions upfront. For a real Lie algebra $\mathfrak{g}$ let us denote by $\mathfrak{g}_{\mathbb{C}}=\mathfrak{g} \otimes_{\mathbb{R}} \mathbb{C}$ its complexification. Likewise, if not stated otherwise, for a connected Lie group $G$ we write $G_{\mathbb{C}}$ for its universal complexification, cf. [Hoc65, $\S$ XVII.5]. If $\varphi: G \rightarrow H$ is a homomorphism of connected Lie groups, then we also denote the following by $\varphi$ :

- the derived homomorphism $d \varphi(\mathbf{1}): \operatorname{Lie}(G) \rightarrow \operatorname{Lie}(H)$;

- the extension of $\varphi$ to a holomorphic homomorphism $G_{\mathbb{C}} \rightarrow H_{\mathbb{C}}$.

Let $G$ be a connected semisimple Lie group with Lie algebra $\mathfrak{g}$. We assume that $G \subset G_{\mathbb{C}}$ and that $G_{\mathbb{C}}$ is simply connected. Let $\tau: G \rightarrow G$ be a non-trivial involution and write $H$ (respectively $H_{\mathbb{C}}$ ), for the $\tau$-fixed points in $G$ (respectively $G_{\mathbb{C}}$ ). The object of concern is the affine symmetric space $X=G / H$. We observe that $X$ is contained in its complexification $X_{\mathbb{C}}=G_{\mathbb{C}} / H_{\mathbb{C}}$ as a totally real submanifold. Write $x_{0}=H_{\mathbb{C}}$ for the base point in $X_{\mathbb{C}}$. 
Let $\mathfrak{h}$ be the Lie algebra of $H$ and note that $\mathfrak{g}=\mathfrak{h}+\mathfrak{q}$ with $\left.\tau\right|_{\mathfrak{q}}=-\mathrm{id}_{\mathfrak{q}}$. The symmetric pair $(\mathfrak{g}, \mathfrak{h})$ is called irreducible if $\mathfrak{g}$ does not contain any $\tau$-invariant ideals except the trivial ideals, $\{0\}$ and $\mathfrak{g}$. In that case, either $\mathfrak{g}$ is simple or $\mathfrak{g}=\mathfrak{g}_{1} \times \mathfrak{g}_{1}$ with $\mathfrak{g}_{1}$ simple and $\tau\left(x, x^{\prime}\right)=\left(x^{\prime}, x\right)$. We say that $X$ is irreducible if $(\mathfrak{g}, \mathfrak{h})$ is irreducible. From now on we assume that $X$ is irreducible.

Fix a Cartan involution $\theta: G \rightarrow G$ commuting with $\tau$. Denote by $K<G$ the subgroup of $\theta$-fixed points and write $Y=G / K$ for the associated Riemannian symmetric space. Write $\mathfrak{k}$ for the Lie algebra of $K$. Then $\mathfrak{g}=\mathfrak{k}+\mathfrak{s}$ with $\left.\theta\right|_{\mathfrak{s}}=-\mathrm{id}_{\mathfrak{s}}$. Note that the universal complexification $K_{\mathbb{C}}$ of $K$ naturally identifies with the $\theta$-fixed points in $G_{\mathbb{C}}$.

We assume that $G$ is a Lie group of Hermitian type, that is, $Y$ is Riemannian symmetric space of Hermitian type. The assumption can be phrased algebraically: $\mathfrak{z}(\mathfrak{k}) \neq\{0\}$ with $\mathfrak{z}(\mathfrak{k})$ the center of $\mathfrak{k}$.

We assume that $\tau$ induces an anti-holomorphic involution on $Y$ and then call $X$ an affine symmetric space of Hermitian type.

Remark 1.1. (a) Our assumptions on $G$ and $\tau$ can be phrased algebraically, namely

$$
\mathfrak{z}(\mathfrak{k}) \cap \mathfrak{q} \neq\{0\} .
$$

Let us mention that another way to formulate (A) is to say that $\mathfrak{q}$ admits an $H$-invariant regular elliptic cone, that is, $X$ is compactly causal [HO96].

(b) Symmetric spaces of Hermitian type resemble compact symmetric spaces on an analytical level. Combined they form the class of symmetric spaces that admit lowest weight modules in their $L^{2}$-spectrum (holomorphic discrete series).

As $X$ is irreducible, it follows that $\mathfrak{z}(\mathfrak{k}) \cap \mathfrak{q}=i \mathbb{R} Z_{0}$ is one dimensional. It is possible to normalize $Z_{0}$ in such a way that the spectrum of $\operatorname{ad}\left(Z_{0}\right)$ is $\{-1,0,1\}$. The zero-eigenspace is $\mathfrak{k}_{\mathbb{C}}$. We denote the +1 -eigenspace in $\mathfrak{s}_{\mathbb{C}}$ by $\mathfrak{s}^{+}$, and the -1-eigenspace by $\mathfrak{s}^{-}$.

Let $\mathfrak{t}$ be a maximal abelian subspace in $\mathfrak{q}$ containing $i Z_{0}$. Then $\mathfrak{t}$ is contained in $\mathfrak{k} \cap \mathfrak{q}$. Set $\mathfrak{a}=i \mathfrak{t}$ and note that $\mathfrak{a}_{\mathbb{C}}=\mathfrak{t}_{\mathbb{C}}$.

Let $\Delta$ be the set of roots of $\mathfrak{t}_{\mathbb{C}}$ in $\mathfrak{g}_{\mathbb{C}}$,

$$
\Delta_{n}=\left\{\alpha \in \Delta \mid \mathfrak{g}_{\mathbb{C}}^{\alpha} \subseteq \mathfrak{s}_{\mathbb{C}}\right\}=\left\{\alpha \in \Delta \mid \alpha\left(Z_{0}\right) \in\{-1,1\}\right\}
$$

and

$$
\Delta_{k}=\left\{\alpha \in \Delta \mid \mathfrak{g}_{\mathbb{C}}^{\alpha} \subseteq \mathfrak{k}_{\mathbb{C}}\right\}=\left\{\alpha \in \Delta \mid \alpha\left(Z_{0}\right)=0\right\} .
$$

Then $\Delta=\Delta_{k} \dot{\cup} \Delta_{n}$. The elements of $\Delta_{n}$ are called non-compact roots and the elements in $\Delta_{k}$ are called compact roots. We choose an ordering in $i t^{*}$ such that $\alpha\left(Z_{0}\right)>0$ implies that $\alpha \in \Delta_{n}^{+} \subseteq \Delta^{+}$. Let $\mathcal{W}$ be the Weyl group of $\Delta$ and $\mathcal{W}_{k}$ the subgroup generated by the reflections coming from the compact roots. As $s\left(Z_{0}\right)=Z_{0}$ for all $s \in \mathcal{W}_{k}$, it follows that $\Delta_{n}^{+}$is $\mathcal{W}_{k}$-invariant.

\subsection{Polyhedrons, cones and the minimal tubes}

Set $A=\exp (\mathfrak{a}), A_{\mathbb{C}}=\exp \left(\mathfrak{a}_{\mathbb{C}}\right), T=\exp (\mathfrak{t})$ and $T_{\mathbb{C}}=\exp \left(\mathfrak{t}_{\mathbb{C}}\right)$. We note that

$$
A_{\mathbb{C}}=T_{\mathbb{C}}=T A \simeq T \times A .
$$

For $\alpha \in \Delta$ let $\check{\alpha} \in \mathfrak{a}$ be its coroot, that is, $\check{\alpha} \in\left[\mathfrak{g}_{\mathbb{C}}^{\alpha}, \mathfrak{g}_{\mathbb{C}}^{-\alpha}\right] \cap \mathfrak{a}$ and $\alpha(\check{\alpha})=2$. Then

$$
\Omega=\sum_{\alpha \in \Delta_{n}^{+}} \mathbb{R}_{>0} \cdot \check{\alpha}
$$

defines a $\mathcal{W}_{k}$-invariant open convex cone in $\mathfrak{a}=i$ that contains $Z_{0}$. Often one refers to $\Omega$ as the minimal cone (it is denoted $c_{\min }$ in [HO96]). Let us remark that one can characterize $\Omega$ as 


\section{S. Gindikin, B. Krötz and G. Ólafsson}

the smallest $\mathcal{W}_{k}$-invariant open convex cone in $\mathfrak{a}$ that contains a long non-compact coroot, that is,

$$
\Omega=\operatorname{co}\left(\mathcal{W}_{k}\left(\mathbb{R}_{>0} \cdot \check{\alpha}\right)\right) \quad\left(\alpha \text { long in } \Delta_{n}^{+}\right) .
$$

Here $\operatorname{co}(\cdot)$ denotes the convex hull of $(\cdot)$.

We set $A_{+}=\exp (\Omega)$ and note that $A_{+} \subset A$ is an open semigroup. Moreover,

$$
\mathcal{T}_{+}=T \exp (\Omega)=T A_{+} \subset T_{\mathbb{C}}
$$

defines a semigroup and complex polyhedron with edge $T$. We also use the notation $A_{-}=\exp (-\Omega)$ and $\mathcal{T}_{-}=T A_{-}$.

Define $G$-invariant subsets of $X_{\mathbb{C}}$ by

$$
D_{ \pm}=G A_{ \pm} \cdot x_{0} \subset X_{\mathbb{C}}
$$

According to [Nee99], $D_{+}$and $D_{-}$are Stein domains in $X_{\mathbb{C}}$ with $X=G \cdot x_{0}$ as Shilov boundary. Subsequently we will refer to $D_{+}$and $D_{-}$as the minimal tube in $X_{\mathbb{C}}$ with edge $X$.

\subsection{Minimal $\overline{\boldsymbol{\theta}} \boldsymbol{\tau}$-stable parabolics}

Denote by $g \mapsto \bar{g}$ the complex conjugation in $G_{\mathbb{C}}$ with respect to the real form $G$. We write $\bar{\theta}$ for the involution on $G_{\mathbb{C}}$ given by $g \mapsto \overline{\theta(g)}$

Let

$$
\mathfrak{n}_{\mathbb{C}}^{+}=\bigoplus_{\alpha \in \Delta_{k}^{+}} \mathfrak{k}_{\mathbb{C}}^{\alpha} \quad \text { and } \quad \mathfrak{n}_{\mathbb{C}}^{-}=\bigoplus_{\alpha \in \Delta_{k}^{+}} \mathfrak{k}_{\mathbb{C}}^{-\alpha}
$$

Set

$$
\begin{gathered}
\mathfrak{n}_{\mathbb{C}}=\mathfrak{n}_{\mathbb{C}}^{+} \oplus \underset{\alpha \in \Delta_{n}^{+}}{\bigoplus} \mathfrak{g}_{\mathbb{C}}^{\alpha}=\mathfrak{n}_{\mathbb{C}}^{+} \oplus \mathfrak{s}^{+}, \\
\mathfrak{m}_{\mathbb{C}}=\left\{U \in \mathfrak{h}_{\mathbb{C}} \mid(\forall V \in \mathfrak{t})[U, V]=0\right\},
\end{gathered}
$$

and

$$
\mathfrak{p}_{\mathbb{C}}=\mathfrak{m}_{\mathbb{C}} \oplus \mathfrak{t}_{\mathbb{C}} \oplus \mathfrak{n}_{\mathbb{C}}
$$

Note that $\mathfrak{m}_{\mathbb{C}}$ is contained in $\mathfrak{k}_{\mathbb{C}}$, as $Z_{0} \in \mathfrak{t}_{\mathbb{C}}$. The Lie algebra $\mathfrak{p}_{\mathbb{C}}$ is a minimal $\bar{\theta} \tau$-stable parabolic subalgebra of $\mathfrak{g}_{\mathbb{C}}$. Define subgroups of $G_{\mathbb{C}}$ by $M_{\mathbb{C}}=Z_{H_{\mathbb{C}}}\left(\mathfrak{t}_{\mathbb{C}}\right) \subset K_{\mathbb{C}}$ and $N_{\mathbb{C}}=\exp \left(\mathfrak{n}_{\mathbb{C}}\right)$.

Note that $T_{\mathbb{C}}=A_{\mathbb{C}}$. Then the prescription

$$
P_{\mathbb{C}}=M_{\mathbb{C}} A_{\mathbb{C}} N_{\mathbb{C}}=M_{\mathbb{C}} T_{\mathbb{C}} N_{\mathbb{C}}
$$

defines a minimal $\bar{\theta} \tau$-stable parabolic subgroup of $G_{\mathbb{C}}$ whose Lie algebra is $\mathfrak{p}_{\mathbb{C}}$. Write $\Gamma=M_{\mathbb{C}} \cap A_{\mathbb{C}}=$ $M \cap T$ and observe that $\Gamma$ is a finite 2-group. The isomorphic map

$$
\left(M_{\mathbb{C}} \times_{\Gamma} A_{\mathbb{C}}\right) \times N_{\mathbb{C}} \rightarrow P_{\mathbb{C}}, \quad([m, a], n) \mapsto \operatorname{man}
$$

yields the structural decomposition of $P_{\mathbb{C}}$.

We denote by $\mathfrak{t} \subseteq \mathfrak{c}$ a $\tau$-stable Cartan subalgebra of $\mathfrak{g}$ contained in $\mathfrak{k}$. Then $\mathfrak{c}=\mathfrak{t} \oplus \mathfrak{c}_{h}$, where $\mathfrak{c}_{h}=\mathfrak{c} \cap \mathfrak{h}$. Denote by $\Sigma$ the set of roots of $\mathfrak{c}_{\mathbb{C}}$ in $\mathfrak{g}_{\mathbb{C}}$. Similarly we set $\Sigma_{n}$, the set of non-compact roots, $\Sigma_{k}$, the set of compact roots. We choose a positive system $\Sigma^{+}$such that $\left.\Sigma^{+}\right|_{\mathfrak{t}} \backslash\{0\}=\Delta^{+}$.

Define tori in $G$ by $C=\exp \mathfrak{c}$ and $C_{h}=\exp \mathfrak{c}_{h}$. We note that $C=T C_{h} \simeq T \times_{\Gamma} C_{h}$.

\section{Complex horospheres I: definition and basic properties}

The objective of this section is to discuss (generic) horospheres on the complex symmetric space

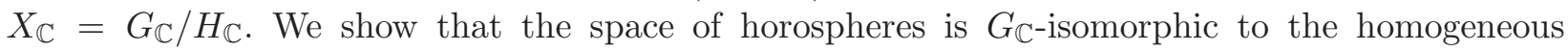


space $\Xi=G_{\mathbb{C}} / M_{\mathbb{C}} N_{\mathbb{C}}$. Further we introduce a $G$-invariant subdomain $\Xi_{+} \subset \Xi$ that is a central object for the rest of this paper.

Write $\xi_{0}=M_{\mathbb{C}} N_{\mathbb{C}}$ for the base point of $\Xi$. Usually we express elements $\xi \in \Xi$ as $\xi=g \cdot \xi_{0}$ for $g \in G_{\mathbb{C}}$.

Consider the following double fibration.

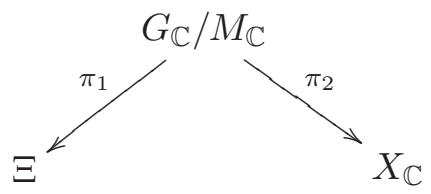

By a horosphere in $X_{\mathbb{C}}$ we mean a subset of the form

$$
E(\xi)=\pi_{2}\left(\pi_{1}^{-1}(\xi)\right) \quad(\xi \in \Xi) .
$$

For $\xi=g \cdot \xi_{0}$, we record that

$$
E(\xi)=g M_{\mathbb{C}} N_{\mathbb{C}} \cdot x_{0}=g N_{\mathbb{C}} \cdot x_{0} \subset X_{\mathbb{C}}
$$

(use $M_{\mathbb{C}} \subset H_{\mathbb{C}}$ ).

Similarly, for $z \in X_{\mathbb{C}}$ we set

$$
S(z)=\pi_{1}\left(\pi_{2}^{-1}(z)\right) .
$$

If $z=g \cdot x_{0}$ for $g \in G_{\mathbb{C}}$, then note that $S(z)=g H_{\mathbb{C}} \cdot \xi_{0}$. Moreover, for $z \in X_{\mathbb{C}}$ and $\xi \in \Xi$ one has the incidence relations

$$
z \in E(\xi) \Longleftrightarrow \pi_{1}^{-1}(\xi) \cap \pi_{2}^{-1}(z) \neq \emptyset \Longleftrightarrow \xi \in S(z) .
$$

The space of horospheres on $X_{\mathbb{C}}$ shall be denoted by $\operatorname{Hor}\left(X_{\mathbb{C}}\right)$, that is,

$$
\operatorname{Hor}\left(X_{\mathbb{C}}\right)=\{E(\xi) \mid \xi \in \Xi\} .
$$

Our first objective is to show that $\Xi$ parameterizes $\operatorname{Hor}\left(X_{\mathbb{C}}\right)$.

Proposition 2.1. The map

$$
E: \Xi \rightarrow \operatorname{Hor}\left(X_{\mathbb{C}}\right), \quad \xi \mapsto E(\xi)
$$

is a $G_{\mathbb{C} \text {-equivariant bijection. }}$

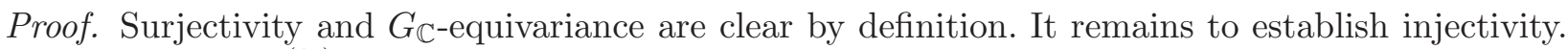
For that write $G_{\mathbb{C}}^{E\left(\xi_{0}\right)}$ for the stabilizer of $E\left(\xi_{0}\right)$ in $G_{\mathbb{C}}$. By $G_{\mathbb{C}^{-} \text {-equivariance it is enough to show that }}$ $G_{\mathbb{C}}^{E\left(\xi_{0}\right)} \subseteq M_{\mathbb{C}} N_{\mathbb{C}}$. Assume that $g \cdot E\left(\xi_{0}\right)=E\left(\xi_{0}\right)$. Then $g N_{\mathbb{C}} \subseteq N_{\mathbb{C}} H_{\mathbb{C}}$. In particular, $g=n h \in N_{\mathbb{C}} H_{\mathbb{C}}$. As $G_{\mathbb{C}}^{E\left(\xi_{0}\right)}$ is a group and $n \in G_{\mathbb{C}}^{E\left(\xi_{0}\right)}$, it follows that $h \in G_{\mathbb{C}}^{E\left(\xi_{0}\right)}$. By Lemma 2.2 (from below) it follows that $h \in M_{\mathbb{C}}$. Hence, $g=h\left(h^{-1} n h\right) \in M_{\mathbb{C}} N_{\mathbb{C}}$, as $M_{\mathbb{C}}$ normalizes $N_{\mathbb{C}}$.

Lemma 2.2. Assume that $h \in H_{\mathbb{C}}$ is such that $h \cdot E\left(\xi_{0}\right)=E\left(\xi_{0}\right)$. Then $h \in M_{\mathbb{C}}$.

Proof. Identify the tangent space $T_{x_{0}}\left(G_{\mathbb{C}} / H_{\mathbb{C}}\right)$ with $\mathfrak{g}_{\mathbb{C}} / \mathfrak{h}_{\mathbb{C}}$. Then, as $\left(h N_{\mathbb{C}} h^{-1}\right) \cdot x_{0}=N_{\mathbb{C}} \cdot x_{0}$, it follows that

$$
\operatorname{Ad}(h)\left(\mathfrak{n}_{\mathbb{C}} \oplus \mathfrak{h}_{\mathbb{C}}\right)=\mathfrak{n}_{\mathbb{C}} \oplus \mathfrak{h}_{\mathbb{C}}
$$

Thus, if $U \in \mathfrak{n}_{\mathbb{C}}$, there exists $Z \in \mathfrak{n}_{\mathbb{C}}$ and $L \in \mathfrak{h}_{\mathbb{C}}$ such that $\operatorname{Ad}(h) U=Z+L$. Applying $(1-\tau)$ to this equality, we get $\operatorname{Ad}(h)(U-\tau(U))=Z-\tau(Z)$. As $\mathfrak{q}_{\mathbb{C}}=(\mathbf{1}-\tau)\left(\mathfrak{n}_{\mathbb{C}}\right) \oplus \mathfrak{t}_{\mathbb{C}}$, and this sum is orthogonal with respect to Killing form, it follows that $\operatorname{Ad}(h) \mathfrak{t}_{\mathbb{C}}=\mathfrak{t}_{\mathbb{C}}$. In particular, $h \in N_{H_{\mathbb{C}}}\left(\mathfrak{t}_{\mathbb{C}}\right)$.

We recall the Riemannian dual $X^{r}=G^{r} / K^{r}$ of $X=G / H$, which corresponds to the Lie algebras $\mathfrak{g}^{r}=\mathfrak{k}^{r}+\mathfrak{s}^{r}$ with $\mathfrak{k}^{r}=(\mathfrak{h} \cap \mathfrak{k})+i(\mathfrak{h} \cap \mathfrak{s})$ and $\mathfrak{s}^{r}=i(\mathfrak{q} \cap \mathfrak{k})+(\mathfrak{q} \cap \mathfrak{s})$. Note that $\mathfrak{a}$ is maximal abelian in $\mathfrak{s}^{r}$. 


\section{S. Gindikin, B. Krötz and G. Ólafsson}

To continue with the proof, we observe that $N_{H_{\mathbb{C}}}\left(\mathfrak{t}_{\mathbb{C}}\right)=N_{K^{r}}(\mathfrak{a}) M_{\mathbb{C}}$. Thus, we may assume that $h \in N_{K^{r}}(\mathfrak{a})$. Write $\sigma_{r}$ for the complex conjugation in $G_{\mathbb{C}}$ with respect to the real form $G^{r}$. Then taking $\sigma^{r}$ fixed points in $h N_{\mathbb{C}} \in N_{\mathbb{C}} H_{\mathbb{C}}$ yields $h N^{r} \in N^{r} K^{r}$ with $N^{r}=G^{r} \cap N_{\mathbb{C}}$. Thus the situation is reduced to the Riemannian case where it follows from [Hel94, p. 78].

It is crucial to observe that there is a $T_{\mathbb{C}}$-action on $\Xi$ that commutes with the left $G_{\mathbb{C}}$ action, as follows.

Proposition 2.3. Let $\xi=g \cdot \xi_{0} \in \Xi, g \in G_{\mathbb{C}}$. For $t \in T_{\mathbb{C}}$ the prescription

$$
\xi \cdot t=g t \cdot \xi_{0}
$$

defines an element of $\Xi$. In particular,

$$
T_{\mathbb{C}} \times \Xi \rightarrow \Xi, \quad(t, \xi) \mapsto \xi \cdot t^{-1}
$$

defines an action of $T_{\mathbb{C}}$ on $\Xi$, which commutes with the natural action of $G$ on $\Xi$.

Proof. As $T_{\mathbb{C}}$ normalizes $M_{\mathbb{C}} N_{\mathbb{C}}$ it follows that (2.5) is defined. Finally, (2.5) implies that (2.6) defines a left action of $T_{\mathbb{C}}$.

It is obvious that the map

$$
\left(G_{\mathbb{C}} \times T_{\mathbb{C}}\right) \times \Xi \rightarrow \Xi, \quad((g, t), \xi) \mapsto g \cdot \xi \cdot t^{-1}
$$

is a holomorphic action of the complex group $G_{\mathbb{C}} \times T_{\mathbb{C}}$ on the homogeneous space $\Xi$.

The remainder of this section is devoted to the definition and basic discussion of an important $G \times T$-invariant subset $\Xi_{+}$of $\Xi$.

We recall from $\S 1.1$ the polydisc $\mathcal{T}_{+}=T A_{+}$and define

$$
\Xi_{+}=G \mathcal{T}_{+} \cdot \xi_{0}=G A_{+} \cdot \xi_{0} .
$$

We record that $\Xi_{+}$is a $(G \times T)$-invariant subset of $\Xi$.

Note that $G / M T$ is the base space of the fiber bundle $G / M \times_{T} \mathcal{T}_{+} \rightarrow G / M T$ with fiber $\mathcal{T}_{+} / \Gamma$. There is a natural action of $G \times T$ on $G / M \times_{T} \mathcal{T}_{+}$given by

$$
(G \times T) \times\left(G / M \times_{T} \mathcal{T}_{+}\right) \rightarrow G / M \times_{T} \mathcal{T}_{+}, \quad((g, t),[x M, a]) \mapsto\left[g x M, a t^{-1}\right] .
$$

The next lemma gives us basic structural information on $\Xi_{+}$.

Lemma 2.4. The set $\Xi_{+}$is open in $\Xi=G_{\mathbb{C}} / M_{\mathbb{C}} N_{\mathbb{C}}$. Moreover, the mapping

$$
\Phi: G / M \times_{T} \mathcal{T}_{+} \rightarrow \Xi, \quad[g M, t] \mapsto g t \cdot \xi_{0}
$$

is a $G \times T$-equivariant diffeomorphism onto $\Xi_{+}$.

Proof. Clearly, $\Phi$ is a defined $G \times T$-equivariant map with $\operatorname{im} \Phi=\Xi_{+}$. Let us show that $\Phi$ is injective. For this assume that $g_{1} t_{1} \cdot \xi_{0}=g_{2} t_{2} \cdot \xi_{0}, g_{j} \in G, t_{j} \in \mathcal{T}_{+}$. By $G$-equivariance we may assume that $g_{2}=\mathbf{1}$. Then $g_{1} \in G \cap P_{\mathbb{C}}=M T$ and without loss of generality we may assume that $g_{1} \in M$. Consequently, as $T_{\mathbb{C}} \cap M_{\mathbb{C}} N_{\mathbb{C}}=\Gamma$, we obtain $t_{1} \in t_{2} \Gamma$, that is, $\left[M, t_{1}\right]=\left[M, t_{2}\right]$. Hence, $\Phi$ is injective.

A standard computation yields that $d \Phi$ is an immersion and a simple dimension count shows that $\operatorname{dim} G / M T+\operatorname{dim} \mathcal{T}_{+}=\operatorname{dim} \Xi$. In particular, $\Phi$ is a submersion and $\operatorname{im} \Phi=\Xi_{+}$is open, concluding the proof of the lemma.

The set $G P_{\mathbb{C}}$ is open in $G_{\mathbb{C}}$ and $G \cap P_{\mathbb{C}}=M T$. Hence, $G / M T$ can be viewed as an open, complex submanifold of the flag manifold $F=G_{\mathbb{C}} / P_{\mathbb{C}}$. We write $F_{+}=G P_{\mathbb{C}} / P_{\mathbb{C}}$ for the image of $G / M T$ in $F$ and call $F_{+}$the flag domain. Although obvious, we emphasize that $F_{+}$is $G$-homogeneous. 
Consider now the following commutative diagram.

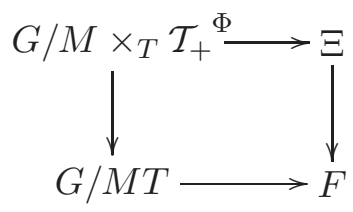

By our preceding remarks and Lemma 2.4, the horizontal lines are real analytic isomorphisms onto open subdomains $\left(\Xi_{+}\right.$and $\left.F_{+}\right)$. Further, the right-hand column constitutes a holomorphic fiber bundle $\Xi \rightarrow F$ with fibers $A_{\mathbb{C}} / \Gamma$. We hence conclude that $G / M \times_{T} \mathcal{T}_{+} \rightarrow G / M T$ is a holomorphic fiber bundle with fiber $\mathcal{T}_{+} / \Gamma$.

\subsection{Fiberings}

To conclude this section we mention three natural fibrations in relation to $\Xi_{+}$and $F_{+}$. Write $S^{+}=\exp \left(\mathfrak{s}^{+}\right)$and recall that the map

$$
Y=G / K \rightarrow G_{\mathbb{C}} / K_{\mathbb{C}} S^{+}, \quad g K \mapsto g K_{\mathbb{C}} S^{+}
$$

is a $G$-equivariant open embedding. Henceforth, $Y$ should be understood as an open subset of the flag manifold $G_{\mathbb{C}} / K_{\mathbb{C}} S^{+}$. We note that $K_{\mathbb{C}} S^{+}$is a maximal parabolic subgroup of $G_{\mathbb{C}}$ that contains $P_{\mathbb{C}}$.

LEMma 2.5. The following assertions hold.

(i) The natural map

$$
\Xi_{+} \rightarrow F_{+}, \quad z M_{\mathbb{C}} N_{\mathbb{C}} \mapsto z P_{\mathbb{C}}
$$

is a holomorphic fibration with fiber $\mathcal{T}_{+} / \Gamma$.

(ii) The restriction of the holomorphic fiber bundle $F \rightarrow G_{\mathbb{C}} / K_{\mathbb{C}} S^{+}, g P_{\mathbb{C}} \mapsto g K_{\mathbb{C}} S^{+}$to the flag domain

$$
F_{+} \rightarrow Y, \quad g M T \rightarrow g K
$$

yields a holomorphic fibration with fiber the flag variety $K / M T \simeq K_{\mathbb{C}} /\left(K_{\mathbb{C}} \cap P_{\mathbb{C}}\right)$.

(iii) The natural map

$$
\Xi_{+} \rightarrow Y, \quad g t \cdot \xi_{0} \mapsto g K
$$

is a holomorphic fibration with fiber $K / M \times_{T} \mathcal{T}_{+}$.

Proof. Assertions (i) and (ii) are clear. Finally, assertion (iii) is a consequence of assertions (i) and (ii).

\section{The $G \times T$-Fréchet module $\mathcal{O}\left(\Xi_{+}\right)$}

The natural action of $G \times T$ on $\Xi_{+}$gives rise to a representation of $G \times T$ on the Fréchet space $\mathcal{O}\left(\Xi_{+}\right)$ of holomorphic functions on $\Xi_{+}$. Our goal is to decompose $\mathcal{O}\left(\Xi_{+}\right)$with respect to this action. By the compactness of $T$, it is clear that $\mathcal{O}\left(\Xi_{+}\right)$decomposes discretely under $T$. It turns out that each $T$-isotypical component is the section module of a holomorphic line bundle over the flag domain $F_{+}$ and that all such section modules arise in this manner.

In the second part of this section, we turn our attention to $G \times T$-invariant Hilbert spaces of holomorphic functions on $\Xi_{+}$. By definition these are unitary $G \times T$-modules $\mathcal{H}$ with continuous $G \times T$-equivariant embeddings into $\mathcal{O}\left(\Xi_{+}\right)$. There are many interesting examples such as weighted Bergman and weighted Hardy spaces. We discuss the Hardy space $\mathcal{H}^{2}\left(\Xi_{+}\right)$on $\Xi_{+}$with constant 


\section{S. Gindikin, B. Krötz and G. Ólafsson}

weight and show that $\mathcal{H}^{2}\left(\Xi_{+}\right)$constitutes a natural model for the $H$-spherical holomorphic discrete series of $G$.

\subsection{The decomposition of $\mathcal{O}\left(\Xi_{+}\right)$}

In $\S 2$, we exhibited a natural action of $G \times T$ on $\Xi_{+}$, namely

$$
(G \times T) \times \Xi_{+} \rightarrow \Xi_{+}, \quad((g, t), \xi) \mapsto g \cdot \xi \cdot t^{-1} .
$$

We recall that $\mathcal{O}\left(\Xi_{+}\right)$becomes a Fréchet space when endowed with the topology of compact convergence.

Remark 3.1. Finite-dimensional representation theory of $G_{\mathbb{C}}$ shows that $\Xi$ (and, hence, $\Xi_{+}$) is holomorphically separable. In particular, $\mathcal{O}\left(\Xi_{+}\right) \neq\{0\}$.

Denote by $\operatorname{GL}\left(\mathcal{O}\left(\Xi_{+}\right)\right)$the group of bounded invertible operators on $\mathcal{O}\left(\Xi_{+}\right)$.

The action (3.1) induces a continuous representation of $G \times T$ on $\mathcal{O}\left(\Xi_{+}\right)$:

$$
L \times R: G \times T \rightarrow \operatorname{GL}\left(\mathcal{O}\left(\Xi_{+}\right)\right), \quad((L \times R)(g, t) f)(\xi)=f\left(g^{-1} \cdot \xi \cdot t\right),
$$

with $(g, t) \in G \times T, f \in \mathcal{O}\left(\Xi_{+}\right)$and $\xi \in \Xi_{+}$.

We first decompose $\mathcal{O}\left(\Xi_{+}\right)$under the action of the compact torus $T$. Denote by $\widehat{T / \Gamma}$ the character group of $T / \Gamma$, that is, $\widehat{T / \Gamma}=\operatorname{Hom}_{\text {cont }}\left(T / \Gamma, \mathbb{S}^{1}\right)$. In the following we identify $\widehat{T / \Gamma}$ with the lattice

$$
\Lambda=\left\{\lambda \in \mathfrak{a}^{*} \mid \forall U \in\left(\left.\exp \right|_{\mathfrak{t}}\right)^{-1}(\Gamma) \lambda(U) \in 2 \pi i \mathbb{Z}\right\} .
$$

Explicitly, one associates the character $\chi_{\lambda}(t \Gamma)=e^{\lambda(\log t)}$ to $\lambda \in \Lambda$. Often we write $t^{\lambda}$ for $\chi_{\lambda}(t \Gamma)$.

The assumption that $G_{\mathbb{C}}$ is simply connected allows an uncomplicated description of the lattice $\Lambda$.

Lemma 3.2. We have

$$
\Lambda=\left\{\lambda \in \mathfrak{a}^{*} \mid(\forall \alpha \in \Delta) \frac{\langle\lambda, \alpha\rangle}{\langle\alpha, \alpha\rangle} \in \mathbb{Z}\right\} .
$$

Proof. (' $\subseteq$ ') Let $\lambda \in \Lambda$. We first show that $\langle\lambda, \alpha\rangle /\langle\alpha, \alpha\rangle \in \mathbb{Z}$ for all $\alpha \in \Delta_{k}$. For that, observe that the compact symmetric space $K / H \cap K$ embeds into $G / H$ via the natural map

$$
K / H \cap K \rightarrow G / H, \quad k(H \cap K) \mapsto k H .
$$

Thus, [Hel84, ch. V, Theorem 4.1] yields that $\langle\lambda, \alpha\rangle /\langle\alpha, \alpha\rangle \in \mathbb{Z}$ for all $\alpha \in \Delta_{k}$. To complete the proof of (' $\subseteq$ ') we still have to verify $\langle\lambda, \alpha\rangle /\langle\alpha, \alpha\rangle \in \mathbb{Z}$ for all $\alpha \in \Delta_{n}$. Fix $\alpha \in \Delta_{n}$. Standard structure theory implies that there is an embedding of symmetric Lie algebras $(\mathfrak{s u}(1,1), \mathfrak{s o}(1,1)) \rightarrow(\mathfrak{g}, \mathfrak{h})$ such that $\left[\begin{array}{cc}i & 0 \\ 0 & -i\end{array}\right] \in \mathfrak{s u}(1,1)$ is mapped to $i \check{\alpha} \in \mathfrak{t}$. As $\mathrm{SL}(2, \mathbb{C})$ is simply connected, we thus obtain an immersive map $\mathrm{SU}(1,1) / \mathrm{SO}(1,1) \rightarrow G / H$. In particular, $\langle\lambda, \alpha\rangle /\langle\alpha, \alpha\rangle \in \mathbb{Z}$ must hold.

(' $\supseteq$ ') Suppose that $\langle\lambda, \alpha\rangle /\langle\alpha, \alpha\rangle \in \mathbb{Z}$ holds for all $\alpha$. Recall the extension $\mathfrak{t} \subseteq \mathfrak{c}$ of $\mathfrak{t}$ to a compact Cartan subalgebra of $\mathfrak{g}$. In the following we consider $\lambda$ as an element of $\mathfrak{c}^{*}$ that is trivial on $\mathfrak{c} \cap \mathfrak{h}$. In [Hel84, p. 537], it was shown that $\lambda$ is analytically integral for $C=\exp \mathfrak{c}$ (this needs that $G_{\mathbb{C}}$ is simply connected). In particular, $\lambda$ defines an element $\chi_{\lambda} \in \hat{T}$. It remains to show that $\left.\chi_{\lambda}\right|_{\Gamma}=\mathbf{1}$. As $M=Z_{H \cap K}(\mathfrak{a})$ and $\Gamma=M \cap T$, this reduces to an assertion on the compact symmetric space $K / H \cap K$, where it follows from [Hel84, ch. V, Theorem 4.1].

For each $\lambda \in \Lambda$, define the $\lambda$-isotypical component of $\mathcal{O}\left(\Xi_{+}\right)$by

$$
\mathcal{O}\left(\Xi_{+}\right)_{\lambda}=\left\{f \in \mathcal{O}\left(\Xi_{+}\right) \mid(\forall t \in T) R(t) f=t^{-\lambda} f\right\} .
$$

As $\left(R, \mathcal{O}\left(\Xi_{+}\right)\right)$is a continuous representation of the compact torus $T$ on a Fréchet space, the PeterWeyl theorem yields

$$
\mathcal{O}\left(\Xi_{+}\right)=\bigoplus_{\lambda \in \Lambda} \mathcal{O}\left(\Xi_{+}\right)_{\lambda}
$$


Each $\mathcal{O}\left(\Xi_{+}\right)_{\lambda}$ is a $G$-module for the representation $L$. In order to describe it explicitly we recall some facts on holomorphic line bundles.

For $\lambda \in \Lambda$, we write $\mathbb{C}_{\lambda}$ for $\mathbb{C}$ when considered as a $M T$-module with trivial $M$-action and $T$ acting by $\chi_{\lambda}$. Recall that $G / M T$ inherits a complex manifold structure through its identification with the flag domain $F_{+}$. In particular, to each $\lambda \in \Lambda$ one associates the holomorphic line bundle

$$
\mathcal{L}_{\lambda}=G \times_{M T} \mathbb{C}_{\lambda} .
$$

Write $\mathcal{O}\left(\mathcal{L}_{\lambda}\right)$ for its $G$-module of holomorphic sections, i.e. $\mathcal{O}\left(\mathcal{L}_{\lambda}\right)$ consists of smooth functions $f: G \rightarrow \mathbb{C}$ such that:

- $f(g m t)=t^{-\lambda} f(g)$ for $g \in G, t \in T$ and $m \in M$;

- $G / M T \rightarrow \mathcal{L}_{\lambda}, g M T \mapsto[g M T, f(g)]$ is holomorphic.

The restriction of $\mathcal{L}_{\lambda}$ to the flag variety $K / M T$ yields the holomorphic line bundle

$$
\mathcal{K}_{\lambda}=K \times_{M T} \mathbb{C}_{\lambda}
$$

over $K / M T$. Write $\Lambda_{0}$ for the $\Delta_{k}^{-}$-dominant elements of $\Lambda$, that is,

$$
\Lambda_{0}=\left\{\lambda \in \Lambda \mid\left(\forall \alpha \in \Delta_{k}^{+}\right)\langle\lambda, \alpha\rangle \leqslant 0\right\} .
$$

According to Bott [Bot57], $V_{\lambda}=\mathcal{O}\left(\mathcal{K}_{\lambda}\right)$ is finite dimensional and non-trivial if and only if $\lambda \in \Lambda_{0}$. By $\mathcal{L}_{\lambda}=G \times_{M T} \mathbb{C}_{\lambda} \simeq G \times_{K}\left(K \times_{M T} \mathbb{C}_{\lambda}\right)$, we retrieve the standard isomorphism

$$
\mathcal{O}\left(\mathcal{L}_{\lambda}\right) \simeq \mathcal{O}\left(G \times_{K} V_{\lambda}\right)
$$

In particular,

$$
\mathcal{O}\left(\mathcal{L}_{\lambda}\right) \neq\{0\} \Longleftrightarrow \lambda \in \Lambda_{0} .
$$

We remind the reader that the $T$-weight spectrum of $\pi_{\lambda}$ is contained in $\lambda+\mathbb{Z}_{\geqslant 0}\left[\Delta^{+}\right]$. In particular, $\mathcal{O}\left(\mathcal{L}_{\lambda}\right)$, if irreducible, is a lowest weight module for $G$ with respect to the positive system $\Delta^{+}$and lowest weight $\lambda$.

Finally, we establish the connection between $\mathcal{O}\left(\Xi_{+}\right)_{\lambda}$ and $\mathcal{O}\left(\mathcal{L}_{\lambda}\right)$. For this, let us denote by $\Xi_{0}$ the pre-image of $F_{+}$in $\Xi$, that is,

$$
\Xi_{0}=G T_{\mathbb{C}} \cdot \xi_{0}
$$

Note that $\Xi_{+} \subset \Xi_{0}$. Holomorphicity and $T$-equivariance yield $\mathcal{O}\left(\Xi_{+}\right)_{\lambda}=\mathcal{O}\left(\Xi_{0}\right)_{\lambda}$. Likewise, restriction yields $\mathcal{O}\left(\Xi_{0}\right)_{\lambda} \simeq \mathcal{O}\left(\mathcal{L}_{\lambda}\right)$. Thus, holomorphic extension and restriction gives a natural $G$-isomorphism $\mathcal{O}\left(\Xi_{+}\right)_{\lambda} \simeq \mathcal{O}\left(\mathcal{L}_{\lambda}\right)$.

We summarize our discussion.

Proposition 3.3. The $G \times T$-Fréchet module $\mathcal{O}\left(\Xi_{+}\right)$decomposes as

$$
\mathcal{O}\left(\Xi_{+}\right)=\bigoplus_{\lambda \in \Lambda_{0}} \mathcal{O}\left(\Xi_{+}\right)_{\lambda}
$$

Moreover, holomorphic extension and restriction canonically identifies $\mathcal{O}\left(\Xi_{+}\right)_{\lambda}$ with the section module $\mathcal{O}\left(\mathcal{L}_{\lambda}\right)$.

We conclude this section with some comments on unitarization of the section modules $\mathcal{O}\left(\mathcal{L}_{\lambda}\right)$.

Remark 3.4. Let $\lambda \in \Lambda_{0}$ and let us denote by $\mathcal{O}\left(\mathcal{L}_{\lambda}\right)^{K \text {-fin }}$ the $(\mathfrak{g}, K)$-module of $K$-finite sections of $\mathcal{O}\left(\mathcal{L}_{\lambda}\right)$. Let us assume that $\mathcal{O}\left(\mathcal{L}_{\lambda}\right)^{K \text {-fin }}$ is irreducible. Then $\mathcal{O}\left(\mathcal{L}_{\lambda}\right)^{K \text {-fin }}$ identifies with the generalized Verma module $N(\lambda)=\mathcal{U}\left(\mathfrak{g}_{\mathbb{C}}\right) \otimes_{\mathcal{U}\left(\mathfrak{k}_{\mathbb{C}}+\mathfrak{s}^{-}\right)} V_{\lambda}$ and the Shapovalov form on $N(\lambda)$ gives rise to the (up to scalar unique) equivariant Hermitian form on $\mathcal{O}\left(\mathcal{L}_{\lambda}\right)^{K \text {-fin }}$. We say that $\mathcal{O}\left(\mathcal{L}_{\lambda}\right)^{K \text {-fin }}$ is unitarizable if the Shapovalov form is positive definite. Another way to formulate it is that there exists a unitary 


\section{S. Gindikin, B. Krötz and G. Ólafsson}

lowest weight representation $\left(\pi_{\lambda}, \mathcal{H}_{\lambda}\right)$ such that the $(\mathfrak{g}, K)$-module of $K$-finite vectors $\mathcal{H}_{\lambda}^{K \text {-fin is }}$ $(\mathfrak{g}, K)$-isomorphic to $\mathcal{O}\left(\mathcal{L}_{\lambda}\right)^{K \text {-fin }}$. In this situation $\mathcal{O}\left(\mathcal{L}_{\lambda}\right)$ is then naturally $G$-isomorphic to the hyperfunction vectors $\mathcal{H}_{\lambda}^{-\omega}$ of $\pi_{\lambda}$.

We want to emphasize that not all $\lambda \in \Lambda_{0}$ correspond to unitarizable modules $\mathcal{O}\left(\mathcal{L}_{\lambda}\right)^{K \text {-fin }}$ (a necessary condition is $\left.\lambda\right|_{\Omega} \geqslant 0$ and we refer to [EHW83] for more precise information). However, we want to stress that $\mathcal{O}\left(\mathcal{L}_{\lambda}\right)^{K \text {-fin }}$ is automatically unitarizable if $\left.\lambda\right|_{\Omega}$ is sufficiently positive (for example, if condition (3.12) below is satisfied).

\subsection{The Hardy space on $\Xi_{+}$}

The objective of this section is to introduce the Hardy space on $\Xi_{+}$and to prove some of its basic properties.

We begin with some measure-theoretic preliminaries. The groups $G_{\mathbb{C}}$ and $M_{\mathbb{C}} N_{\mathbb{C}}$ are unimodular

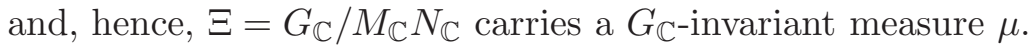

Recall that $M$ is a compact subgroup of $G$ and denote by $d m$ a normalized Haar measure on $M$. Further, we let $d g$ and $d(g M)$ denote left $G$-invariant measures on $G$ (respectively $G / M$ ), normalized by the condition

$$
\int_{G} f(g) d g=\int_{G / M} \int_{M} f(g m) d m d(g M)
$$

for all $f \in L^{1}(G)$.

Note that the stabilizer in $G$ of any point $\xi \in \mathcal{T}_{+} \cdot \xi_{0} \subset \Xi_{+}$is the compact subgroup $M$. In particular, one has

$$
\int_{G} f(g \cdot \xi) d g=\int_{G / M} f(g \cdot \xi) d(g M)
$$

for all $\xi \in \mathcal{T}_{+} \cdot \xi_{0}$ and integrable functions $f$ on $\Xi_{+}$.

Write $\|\cdot\|_{2}$ for the $L^{2}$-norm on $L^{2}(G)$. Let us remark that the representation $\left(R, \mathcal{O}\left(\Xi_{+}\right)\right)$of $T$ naturally extends to a representation of the semigroup $\mathcal{T}_{-} \cup T$, also denoted by $R$. Furthermore, if $f \in \mathcal{O}\left(\Xi_{+}\right)$and $t \in \mathcal{T}_{-}$, then we can define the restriction of $R(t) f$ to $G$ by $\left.R(t) f\right|_{G}: G \rightarrow \mathbb{C}$ by $\left.R(t) f\right|_{G}(g)=f\left(g t^{-1} \cdot \xi_{0}\right)$. The Hardy norm of $f \in \mathcal{O}\left(\Xi_{+}\right)$is defined by

$$
\|f\|^{2}=\sup _{t \in \mathcal{T}_{+}} \int_{G}\left|f\left(g t \cdot \xi_{0}\right)\right|^{2} d g=\sup _{t \in \mathcal{T}_{-}}\left\|\left.R(t) f\right|_{G}\right\|_{2}^{2} .
$$

Let

Obviously

$$
\mathcal{H}^{2}\left(\Xi_{+}\right)=\left\{f \in \mathcal{O}\left(\Xi_{+}\right) \mid\|f\|<\infty\right\}
$$

$$
\|R(t) f\| \leqslant\|f\| \quad \text { for all } t \in \mathcal{T}_{-}
$$

and, hence, $\mathcal{T}_{-}$acts on $\mathcal{H}^{2}\left(\Xi_{+}\right)$by contractions. Note that $\left.R(t) f\right|_{G}$ is right $M$-invariant and, by the definition of the Hardy space norm, $\left.R(t) f\right|_{G} \in L^{2}(G / M) \subseteq L^{2}(G)$.

Lemma 3.5. The space $\mathcal{H}^{2}\left(\Xi_{+}\right)$is a Hilbert space. Furthermore, the following hold.

(i) For $\xi \in \Xi_{+}$the point evaluation map ev $\xi: \mathcal{H}^{2}\left(\Xi_{+}\right) \ni f \mapsto f(\xi) \in \mathbb{C}$ is continuous.

(ii) The boundary value map $\beta: \mathcal{H}^{2}\left(\Xi_{+}\right) \rightarrow L^{2}(G / M) \subseteq L^{2}(G)$

$$
\beta(f)=\left.\lim _{\mathcal{T}_{-} \ni t \rightarrow e} R(t) f\right|_{G}
$$

is an equivariant isometry into $L^{2}(G / M)$.

Proof. The proof follows a standard procedure and is more a sketch. We refer to [HOO91], in particular the proof of Theorem 2.2, for a detailed discussion of the underlying methods. 
Let $\xi \in \Xi_{+}$. Then there exist relatively compact open sets $U_{G} \subseteq G$ and $U_{T} \subseteq \mathcal{T}_{+}$such that $\xi \in U_{G} U_{T} \cdot \xi_{0}$. Thus, there is a constant $c>0$ such that the Bergman-type estimate

$$
\int_{U_{G} U_{T} \cdot \xi_{0}}|f(\xi)|^{2} d \mu(\xi) \leqslant c \cdot\|f\|^{2}
$$

holds for all $f \in \mathcal{H}^{2}\left(\Xi_{+}\right)$. This implies that $\mathcal{H}^{2}\left(\Xi_{+}\right)$is complete, and that point evaluations are continuous.

Write $\mathbb{C}_{+}=\{z \in \mathbb{C} \mid \operatorname{Im}(z)>0\}$ for the upper half-plane and fix $Z \in i \Omega$. We note that the map $\left.\mathcal{T}_{-} \ni t \mapsto R(t) f\right|_{G} \in L^{2}(G)$ is well defined and holomorphic. Hence,

$$
L_{f}: \mathbb{C}_{+} \rightarrow L^{2}(G / M) ; L_{f}(z)=\left.R(\exp (z Z)) f\right|_{G} \in L^{2}(G / M)
$$

defines a holomorphic function on $\mathbb{C}_{+}$.

By [HOO91, Lemma 2.3] it follows that $\lim _{z \rightarrow 0} L_{f}(z)$ exists and is monotonically increasing as $s \searrow 0$ along each line segment $\exp ($ siZ $)$ or, because of the right invariance of $d g$, on each $t \exp (\operatorname{siZ})$, $t \in T$. As in [HOO91], we show that this limit is independent of $Z$. Thus, we get a boundary value $\operatorname{map} \beta: \mathcal{H}^{2}\left(\Xi_{+}\right) \rightarrow L^{2}(G / M)$, defined by

$$
\beta(f)=\left.\lim _{t \rightarrow e} R(t) f\right|_{G} .
$$

By the definition of the Hardy space norm, we obviously have

$$
\|\beta(f)\|_{2} \leqslant\|f\| \text {. }
$$

However, as the norm $\|R(\exp (s Z)) f\|_{2}$ is monotonically increasing for $s \searrow 0$, it follows that

$$
\|R(\exp (s Z)) f\|_{2} \leqslant\|\beta(f)\|
$$

for all $s \in \mathbb{R}^{+}$. Thus,

$$
\left\|\left.R(t) f\right|_{G}\right\| \leqslant\|\beta(f)\| .
$$

It follows that $\beta: \mathcal{H}^{2}\left(\Xi_{+}\right) \rightarrow L^{2}(G)$ is an isometry and, hence, $\mathcal{H}^{2}\left(\Xi_{+}\right)$is a Hilbert space.

Clearly $L \times R$ defines a unitary representation of $G \times T$ on $\mathcal{H}^{2}\left(\Xi_{+}\right)$. Our aim is to decompose $\mathcal{H}^{2}\left(\Xi_{+}\right)$with respect to this action. As before we begin with the decomposition under $T$. For $\lambda \in \Lambda$, the $\lambda$-isotypical component of $\mathcal{H}^{2}\left(\Xi_{+}\right)$is given by $\mathcal{H}^{2}\left(\Xi_{+}\right)_{\lambda}=\mathcal{H}^{2}\left(\Xi_{+}\right) \cap \mathcal{O}\left(\Xi_{+}\right)_{\lambda}$. The Peter-Weyl theorem yields the orthogonal decomposition

$$
\mathcal{H}^{2}\left(\Xi_{+}\right)=\bigoplus_{\lambda \in \Lambda_{0}} \mathcal{H}^{2}\left(\Xi_{+}\right)_{\lambda}
$$

of $\mathcal{H}^{2}\left(\Xi_{+}\right)$in $G$-modules.

We direct our attention to the unitary $G$-modules $\mathcal{H}^{2}\left(\Xi_{+}\right)_{\lambda}$ inside of $\mathcal{O}\left(\Xi_{+}\right)_{\lambda}$.

Suppose that $\mathcal{H}^{2}\left(\Xi_{+}\right)_{\lambda} \neq\{0\}$. Then $\mathcal{O}\left(\mathcal{L}_{\lambda}\right) \neq\{0\}$ and the restriction mapping

$$
\mathcal{H}^{2}\left(\Xi_{+}\right)_{\lambda} \rightarrow \mathcal{O}\left(\mathcal{L}_{\lambda}\right)
$$

gives a $G$-equivariant embedding. Moreover, $\beta\left(\mathcal{H}^{2}\left(\Xi_{+}\right)_{\lambda}\right) \subset L^{2}(G)$. Thus, $\mathcal{H}^{2}\left(\Xi_{+}\right)_{\lambda}$ is a module of the holomorphic discrete series of $G$. In terms of $\lambda$ this means that $\lambda$ satisfies the Harish-Chandra condition [Har56]

where $\rho(\mathfrak{c})=\frac{1}{2} \sum_{\alpha \in \Sigma^{+}} \alpha$.

$$
\langle\lambda-\rho(\mathfrak{c}), \alpha\rangle>0 \quad\left(\forall \alpha \in \Sigma_{n}^{+}\right)
$$

Write $\Lambda_{\text {sd }}$ for the set of all $\lambda \in \Lambda_{0}$ that satisfy (3.12).

Conversely, let $\lambda \in \Lambda_{\text {sd }}$ and write $\mathcal{H}_{\lambda}$ for a corresponding unitary lowest weight module with lowest weight $\lambda$. Denote by $v_{\lambda} \in \mathcal{H}_{\lambda}$ a normalized lowest weight vector and write $d(\lambda)$ for the formal 


\section{S. Gindikin, B. Krötz and G. Ólafsson}

dimension (see [Har56] or (5.13) below). It is then straightforward that

$$
\mathcal{H}_{\lambda} \rightarrow \mathcal{H}^{2}\left(\Xi_{+}\right), \quad v \mapsto\left(g t \cdot \xi_{0} \mapsto \sqrt{d(\lambda)} \cdot t^{-\lambda}\left\langle\pi_{\lambda}\left(g^{-1}\right) v, v_{\lambda}\right\rangle\right)
$$

defines a $G$-equivariant isometric embedding. Hence $\mathcal{H}^{2}\left(\Xi_{+}\right)_{\lambda} \simeq \mathcal{H}_{\lambda} \neq\{0\}$.

Summarizing our discussion we obtain the Plancherel decomposition for $\mathcal{H}^{2}\left(\Xi_{+}\right)$.

Proposition 3.6. As a G-module, the Hardy space decomposes as

$$
\mathcal{H}^{2}\left(\Xi_{+}\right) \simeq \bigoplus_{\lambda \in \Lambda_{\mathrm{sd}}} \mathcal{H}_{\lambda}
$$

Remark 3.7. (a) The set $\Lambda_{\text {sd }}$ describes the set of all $H$-spherical unitary lowest weight representations (up to equivalence) whose matrix coefficients are square integrable on $G$, that is, $\Lambda_{\text {sd }}$ is the spectrum of the $H$-spherical holomorphic discrete series of $G$.

(b) Later we mainly deal with the spectrum $\Lambda_{2}$ of the holomorphic discrete series on $X$. One has

$$
\Lambda_{2} \subseteq \Lambda_{\mathrm{sd}}
$$

with equality precisely for the equal rank cases, that is, $\operatorname{rank}(G)=\operatorname{rank}(G / H)$ (see [OO88, OO91]).

\section{Complex horospheres II: horospheres with no real points}

We continue our discussion of complex horospheres from $\S 2$. We introduce the notion of horosphere without real points and investigate $\Xi_{+}$with respect to this property. In addition, we prove some dual statements for the minimal tubes $D_{ \pm}$.

Definition 4.1. We say that the complex horosphere $E(\xi) \subset X_{\mathbb{C}}$ has no real points if $E(\xi) \cap X=\emptyset$. We denote by $\Xi_{n r} \subset \Xi$ the subset of those $\xi$ that correspond to horospheres with no real points.

Lemma 4.2. The set $\Xi_{n r}$ is a $G$-invariant subset of $\Xi$.

Proof. Let $\xi \in \Xi_{n r}$ and $g \in G$. Assume that $x \in E(g \cdot \xi) \cap X$. Then $g^{-1} x \in E(\xi) \cap X$, contradicting the assumption that $E(\xi)$ has no real points.

Recall the open $G$-invariant subset $\Xi_{+}=G A_{+} \cdot \xi_{0} \subset \Xi$. In the following it will be useful to consider with $\Xi_{+}$its pre-image $\widetilde{\Xi}_{+}$in $G_{\mathbb{C}}$, that is,

$$
\widetilde{\Xi}_{+}=G A_{+} M_{\mathbb{C}} N_{\mathbb{C}}
$$

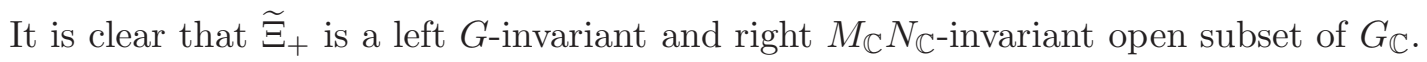

Next we direct our attention to the Zariski open subset $N_{\mathbb{C}} A_{\mathbb{C}} H_{\mathbb{C}}$ of $G_{\mathbb{C}}$. Our objective is to study $\widetilde{\Xi}_{+}$in relation to $N_{\mathbb{C}} A_{\mathbb{C}} H_{\mathbb{C}}$.

Remark 4.3. Note that $\widetilde{\Xi}_{+}^{-1} \subset N_{\mathbb{C}} A_{\mathbb{C}} H_{\mathbb{C}}$ is equivalent to $G \subset N_{\mathbb{C}} A_{\mathbb{C}} H_{\mathbb{C}}$. However, the latter is true only for rank $X=1$, that is $\operatorname{dim} \mathfrak{t}=1$. In general, $G \cap N_{\mathbb{C}} A_{\mathbb{C}} H_{\mathbb{C}}$ is an open and dense subset of $G$ (cf. Theorem 4.5 below).

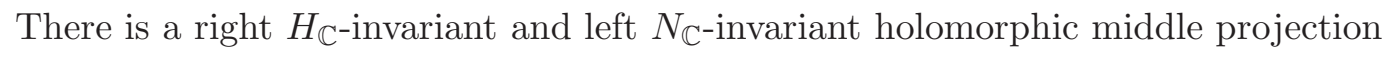

$$
a_{H}: N_{\mathbb{C}} A_{\mathbb{C}} H_{\mathbb{C}} \rightarrow A_{\mathbb{C}} / \Gamma, \quad z \mapsto a_{H}(x)
$$

In particular, for each $\lambda \in \Lambda$ we obtain a natural $\left(N_{\mathbb{C}}, H_{\mathbb{C}}\right)$-invariant holomorphic maps

$$
N_{\mathbb{C}} A_{\mathbb{C}} H_{\mathbb{C}} \rightarrow \mathbb{C}, \quad x \mapsto a_{H}(x)^{\lambda} .
$$

The holomorphic function $N_{\mathbb{C}} A_{\mathbb{C}} \cdot x_{0} \rightarrow A_{\mathbb{C}} / \Gamma$ induced by $a_{H}$ is also denoted by $a_{H}$.

The function $a_{H}$ enables us to give a useful geometric description of horospheres. 
Lemma 4.4. Let $\xi=g \cdot \xi_{0} \in \Xi$ for $g \in G_{\mathbb{C}}$. Then

$$
\begin{aligned}
E(\xi) & =\left\{z \in X_{\mathbb{C}} \mid g^{-1} z \in N_{\mathbb{C}} A_{\mathbb{C}} \cdot x_{0}, a_{H}\left(g^{-1} z\right)=\Gamma\right\} \\
& =\left\{z \in X_{\mathbb{C}} \mid g^{-1} z \in N_{\mathbb{C}} A_{\mathbb{C}} \cdot x_{0}, a_{H}\left(g^{-1} z\right)^{\lambda}=1 \text { for all } \lambda \in \Lambda\right\} .
\end{aligned}
$$

Proof. (' $\subseteq$ ') If $z \in E(\xi)$, then $z=g n \cdot x_{o}$ for some $n \in N_{\mathbb{C}}$. Thus, $g^{-1} z=n \cdot \xi_{0} \in N_{\mathbb{C}} A_{\mathbb{C}} \cdot x_{0}$ and $a_{H}\left(g^{-1} z\right)=a_{H}\left(n \cdot x_{0}\right)=\Gamma$.

(' $\supseteq$ ') Conversely, let $z \in X_{\mathbb{C}}$ be such that $g^{-1} z \in N_{\mathbb{C}} A_{\mathbb{C}} \cdot x_{0}$ and $a_{H}\left(g^{-1} z\right)=\Gamma$. From the first condition, it follows that $g^{-1} z=n a \cdot x_{0}$ for some $n \in N_{\mathbb{C}}$ and $a \in A_{\mathbb{C}}$; the second condition implies $a \in \Gamma$. Thus, $z \in g \cdot \xi_{0}$, as was required.

We define a subset of $\Lambda_{0}$ by

$$
\begin{aligned}
\Lambda_{\geqslant 0} & =\left\{\lambda \in \Lambda_{0}|\lambda|_{\Omega} \geqslant 0\right\} \\
& =\left\{\lambda \in \Lambda|\lambda|_{\Omega} \geqslant 0,\left(\forall \alpha \in \Delta_{k}^{+}\right)\langle\lambda, \alpha\rangle \leqslant 0\right\} .
\end{aligned}
$$

The following theorem is the main geometric result of the paper.

THEOREM 4.5. The following assertions hold.

(i) $G \cap N_{\mathbb{C}} A_{\mathbb{C}} H_{\mathbb{C}}$ is open and dense in $G$.

(ii) Let $\lambda \in \Lambda_{\geqslant 0}$. Then the function $\left.a_{H}^{\lambda}\right|_{G \cap N_{\mathbb{C}} A_{\mathbb{C}} H_{\mathbb{C}}}$ extends to a continuous function on $G$ and

$$
\left|a_{H}(g)^{\lambda}\right| \leqslant 1 \quad(g \in G) .
$$

Proof. The approach to prove this theorem lies in the use of the structural decomposition

$$
G=K A_{q} H
$$

where $A_{q}=\exp \left(\mathfrak{a}_{q}\right)$ with $\mathfrak{a}_{q} \subseteq \mathfrak{s} \cap \mathfrak{q}$ a maximal abelian subspace. There is a natural way to construct such a $\mathfrak{a}_{q}$ out of the weight space decomposition $\mathfrak{g}_{\mathbb{C}}=\mathfrak{a}_{\mathbb{C}}+\mathfrak{m}_{\mathbb{C}}+\bigoplus_{\alpha \in \Delta} \mathfrak{g}_{\mathbb{C}}^{\alpha}$. This is briefly reviewed. Let $\gamma_{1}, \ldots, \gamma_{r} \in \Delta_{n}^{+}$be a maximal set of long strongly orthogonal roots. Then one can find $Z_{j} \in \mathfrak{g}_{\mathbb{C}}^{\gamma_{j}}$, $j=1, \ldots, r$, such that

$$
\mathfrak{a}_{q}=\bigoplus_{j=1}^{r} \mathbb{R}\left(Z_{j}-\tau\left(Z_{j}\right)\right)
$$

is a maximal abelian subspace of $\mathfrak{s} \cap \mathfrak{q}$; further

$$
A_{q} \subset S^{+} \overline{A_{-}} H_{\mathbb{C}}
$$

(see [HO96, pp. 210-211]).

(i) As $S^{+} \subseteq N_{\mathbb{C}}$, we obtain from (4.3) and (4.5) that

$$
G \subset N_{\mathbb{C}} K \overline{A_{-}} H_{\mathbb{C}}
$$

Hence, it is sufficient to show that

$$
K \overline{A_{-}} \cap N_{\mathbb{C}}^{+} A_{\mathbb{C}}\left(H_{\mathbb{C}} \cap K_{\mathbb{C}}\right) \quad \text { is open and dense in } K \overline{A_{-}} .
$$

To continue, we first have to recall some facts related to the Iwasawa decomposition of $K_{\mathbb{C}}$. Write $\widetilde{N}_{\mathbb{C}}^{+}$for a maximal $C$-stable unipotent subgroup of $K_{\mathbb{C}}$ containing $N_{\mathbb{C}}^{+}$and set $\widetilde{A}=\exp (i \mathfrak{c})$. Then $K_{\mathbb{C}}=\widetilde{N}_{\mathbb{C}}^{+} \widetilde{A} K$ is an Iwasawa decomposition of $K_{\mathbb{C}}$. We recall that $\Omega$ and, hence, $\overline{A_{-}}$are $\mathcal{W}_{k^{-}}$ invariant. Thus, Kostant's nonlinear convexity theorem (cf. [Hel84, ch. IV, Theorem 10.5]) implies that $K \overline{A_{-}} \subset \tilde{N}_{\mathbb{C}}^{+} \overline{A_{-}} K$. As $\widetilde{N}_{\mathbb{C}}^{+} \subset N_{\mathbb{C}}^{+} M_{\mathbb{C}}$ and $A \subseteq \widetilde{A} \subseteq A M_{\mathbb{C}}$, we thus get $K \overline{A_{-}} \subset N_{\mathbb{C}}^{+} \overline{A_{-}} M_{\mathbb{C}} K$. In particular, in order to establish (4.6) it is enough to verify that $K \cap N_{\mathbb{C}}^{+} A_{\mathbb{C}}\left(H_{\mathbb{C}} \cap K_{\mathbb{C}}\right)$ is dense in $K$. However, this is known (for example, it follows from [Cle88, Lemme 2.1]). 


\section{S. Gindikin, B. Krötz And G. Ólafsson}

(ii) In the proof of assertion (i) we have seen that $G \subset N_{\mathbb{C}} M_{\mathbb{C}} \overline{A_{-}} K H_{\mathbb{C}}$. Thus, we only have to show that $a_{H}^{\lambda}$ can be defined as a holomorphic function on $K_{\mathbb{C}}$ with $\left|a_{H}(a k)^{\lambda}\right| \leqslant 1$ for all $k \in K$ and $a \in \overline{A_{-}}$. For that let $\left(\tau_{\lambda}, V_{\lambda}\right)$ denote the holomorphic $\left(H_{\mathbb{C}} \cap K_{\mathbb{C}}\right)$-spherical representation of $K_{\mathbb{C}}$ with lowest weight $\lambda$. Write $(\cdot, \cdot)$ for a $K$-invariant inner product on $V_{\lambda}$. Let $v_{\lambda}$ be a normalized lowest weight vector and $v_{H}$ be the spherical vector with $\left(v_{H}, v_{\lambda}\right)=1$. Then, for all $x \in N_{\mathbb{C}}^{+} A_{\mathbb{C}}\left(H_{\mathbb{C}} \cap K_{\mathbb{C}}\right) \subset K_{\mathbb{C}}$, we have

$$
\left(\pi_{\lambda}(x) v_{H}, v_{\lambda}\right)=a_{H}(x)^{\lambda} .
$$

As the left-hand side has a holomorphic extension to $K_{\mathbb{C}}$, the same holds for $a_{H}^{\lambda}$. Finally, for $a \in \overline{A_{-}}$ and $k \in K$, we have

$$
a_{H}(a k)^{\lambda}=a^{\lambda} a_{H}(k)^{\lambda} .
$$

Observe that $a^{\lambda} \leqslant 1$ as $\lambda \in \Lambda_{\geqslant 0}$ and that $\left|a_{H}(k)^{\lambda}\right| \leqslant 1$ for all $k \in K$ by [Cle88, Lemme 2.3] (see also [Osh89]). This completes the proof of assertion (ii).

Theorem 4.5 features interesting and important corollaries.

COROLlary 4.6. Let $\lambda \in \Lambda_{\geqslant 0}$ be such that $\left.\lambda\right|_{\Omega}>0$. Then $\left.a_{H}^{\lambda}\right|_{\widetilde{\Xi}_{+}^{-1} \cap N_{\mathbb{C}} A_{\mathbb{C}} H_{\mathbb{C}}}$ extends to a holomorphic function on $\widetilde{\Xi}_{+}^{-1}$ with

$$
\left|a_{H}(x)^{\lambda}\right|<1 \quad\left(x \in \widetilde{\Xi}_{+}^{-1}\right) .
$$

Corollary 4.7. We have $\Xi_{+} \subseteq \Xi_{n r}$, that is, $E(\xi) \cap X=\emptyset$ for all $\xi \in \Xi_{+}$.

Proof. Suppose that there exists $\xi \in \Xi_{+}$such that $E(\xi) \cap X \neq \emptyset$. Then $\widetilde{\Xi}_{+} \cap H_{\mathbb{C}} \neq \emptyset$, which is equivalent to $\widetilde{\Xi}_{+}^{-1} \cap H_{\mathbb{C}} \neq \emptyset$; a contradiction to the previous corollary.

Remark 4.8 (Monotonicity/convexity). Theorem 4.5(ii) has a natural interpretation in terms of convexity/monotonicity. Write $\operatorname{pr}_{\mathfrak{a}}=\Im \log a_{H}$ and note that $\mathrm{pr}_{\mathfrak{a}}: N_{\mathbb{C}} A_{\mathbb{C}} H_{\mathbb{C}} \rightarrow \mathfrak{a}$ is a well-defined continuous map. Theorem 4.5(ii) is then equivalent to the inclusion

$$
\operatorname{pr}_{\mathfrak{a}}\left(G \cap N_{\mathbb{C}} A_{\mathbb{C}} H_{\mathbb{C}}\right) \subseteq \bigoplus_{\alpha \in \Delta_{n}^{-} \cup \Delta_{k}^{+}} \mathbb{R}_{\geqslant 0} \cdot \check{\alpha}
$$

\subsection{Dual statements for the minimal tubes}

Recall from $\S 1.1$ the minimal tubes $D_{ \pm}=G A_{ \pm} \cdot x_{0}$ in $X_{\mathbb{C}}$ with edge $X$.

It follows from Neeb's nonlinear convexity theorem [Nee94] that

$$
G A_{-} \subseteq N_{\mathbb{C}} M_{\mathbb{C}} A_{-} G .
$$

This fact combined with Theorem 4.5 yields

$$
G A_{-} H_{\mathbb{C}} \cap N_{\mathbb{C}} A_{\mathbb{C}} H_{\mathbb{C}} \subseteq N_{\mathbb{C}} T A_{-} \exp \left(\bigoplus_{\alpha \in \Delta_{k}^{+}} \mathbb{R}_{\geqslant 0} \cdot \check{\alpha}\right) H_{\mathbb{C}} .
$$

We have shown the following.

Corollary 4.9. Let $\lambda \in \Lambda_{\geqslant 0}$ be such that $\left.\lambda\right|_{\Omega}>0$. Then, $\left.a_{H}^{\lambda}\right|_{D_{-} \cap N_{\mathbb{C}} A_{\mathbb{C}} \cdot x_{0}}$ extends to a holomorphic function on $D_{-}$such that

$$
\left|a_{H}(x)^{\lambda}\right|<1 \quad\left(x \in D_{-}\right)
$$

We recall the definition of the orbits $S(z) \subset \Xi$ for $z \in X_{\mathbb{C}}$ (cf. (2.3)). The convexity inclusion (4.9) delivers the dual statement to Corollary 4.7.

Corollary 4.10. We have $S(z) \cap G / M=\emptyset$ for all $z \in D_{-}$. 
Proof. Let $z=g a \cdot x_{0}$ for $g \in G$ and $a \in A_{-}$. Suppose that $S(z) \cap G / M \neq \emptyset$. As $S(z)=g a H_{\mathbb{C}} \cdot \xi_{0}$, this is equivalent to $a H_{\mathbb{C}} N_{\mathbb{C}} \cap G \neq \emptyset$. In other words, $G a \cap N_{\mathbb{C}} H_{\mathbb{C}} \neq \emptyset$; a contradiction to (4.9).

Remark 4.11. Note that (4.8) is equivalent to $A_{+} G \subseteq G A_{+} M_{\mathbb{C}} N_{\mathbb{C}}$. This inclusion exhibits an interesting additional structure of $\Xi_{+}$: it implies

$$
\Xi_{+}=G A_{+} G \cdot \xi_{0}
$$

Remark 4.12 (Generalization to other cones). Let $\tilde{\Omega}$ be a $\mathcal{W}_{k}$-invariant convex open sharp cone in $\mathfrak{a}$ containing $\Omega$. A particular interesting example is the maximal cone (denoted by $c_{\max }$ in [HO96]). In this context we would like to mention that the results in this section remain true for $\Omega$ replaced by $\tilde{\Omega}$, the obvious adjustment of $\Lambda_{\geqslant 0}$ understood.

\section{The horospherical Cauchy transform}

Our geometric results from $\S 4$ enable us to define a natural horospherical Cauchy kernel on $\Xi_{+}$. The kernel gives rise to the horospherical Cauchy transform $L^{1}(X) \rightarrow \mathcal{O}\left(\Xi_{+}\right)$. The main result is a geometric inversion formula for the horospherical Cauchy transform for functions in the holomorphic discrete series on $X$.

\subsection{The horospherical Cauchy kernel}

In this section we define the horospherical Cauchy kernel and the corresponding horospherical Cauchy transform. We introduce the holomorphic spherical Fourier transform and relate it to the horospherical Cauchy transform.

To begin with, we have to recall some features of the root system $\Delta$. Let us denote by

$$
\Pi=\left\{\alpha_{1}, \ldots, \alpha_{m}\right\}
$$

a basis of $\Delta$ corresponding to the positive system $\Delta_{n}^{+} \cup \Delta_{k}^{-}$. As $\operatorname{Spec} \operatorname{ad}\left(Z_{0}\right)=\{-1,0,1\}$, it follows that exactly one member of $\Pi$ is non-compact, say $\alpha_{m}$. Define weights $\omega_{1}, \ldots, \omega_{m} \in \mathfrak{a}^{*}$ by

$$
\frac{\left\langle\omega_{i}, \alpha_{j}\right\rangle}{\left\langle\alpha_{j}, \alpha_{j}\right\rangle}=\delta_{i j} \quad(1 \leqslant i, j \leqslant n)
$$

Set

$$
\Lambda_{>0}=\mathbb{Z}_{\geqslant 0} \cdot \omega_{1}+\cdots+\mathbb{Z}_{\geqslant 0} \cdot \omega_{m-1}+\mathbb{Z}_{>0} \cdot \omega_{m} .
$$

Recall the definition of $\Lambda_{\geqslant 0}$ from (4.1) and (4.2).

LEMma 5.1. The following assertions hold.

(i) $\left.\omega_{i}\right|_{\Omega}>0$ for all $1 \leqslant i \leqslant n$. In particular, $\left.\lambda\right|_{\Omega}>0$ for all $\lambda \in \Lambda_{>0}$.

(ii) $\Lambda_{\geqslant 0}=\mathbb{Z}_{\geqslant 0} \cdot \omega_{1}+\cdots+\mathbb{Z}_{\geqslant 0} \cdot \omega_{m}$. In particular, $\Lambda_{>0} \subset \Lambda_{\geqslant 0}$.

Proof. (i) Fix $x \in \Omega$. Then $x=\sum_{\alpha \in \Delta_{n}^{+}} k_{\alpha} \check{\alpha}$ with $k_{\alpha}>0$. Now each $\alpha \in \Delta_{n}^{+}$can be uniquely expressed as $\alpha=\alpha_{m}+\gamma$ with $\gamma \in \mathbb{Z}_{\geqslant 0}\left[\Delta_{k}^{-}\right]$. Moreover, if $\alpha=\beta$ is the highest root, then $\gamma \in$ $\mathbb{Z}_{>0}\left[\alpha_{1}, \ldots, \alpha_{n-1}\right]$. As $k_{\beta}>0$, the assertion follows.

(ii) Set $\Lambda_{\geqslant 0}^{\prime}=\mathbb{Z}_{\geqslant 0} \cdot \omega_{1}+\cdots+\mathbb{Z}_{\geqslant 0} \cdot \omega_{m}$. We first show that $\Lambda_{\geqslant 0}^{\prime} \subseteq \Lambda_{\geqslant 0}$. For that let $\lambda \in \Lambda_{\geqslant 0}^{\prime}$, say $\lambda=\sum_{i=1}^{m} k_{i} \omega_{i}$ with $k_{i} \in \mathbb{Z}_{\geqslant 0}$. As $\alpha_{1}, \ldots, \alpha_{n-1}$ constitutes a basis of $\Delta_{k}^{-}$, it follows that $\langle\lambda, \alpha\rangle /\langle\alpha, \alpha\rangle \in \mathbb{Z}_{\leqslant 0}$ for all $\alpha \in \Delta_{k}^{+}$. Furthermore, $\left.\lambda\right|_{\Omega} \geqslant 0$ by assertion (i). Hence, $\Lambda_{\geqslant 0}^{\prime} \subseteq \Lambda_{\geqslant 0}$.

Finally, we establish $\Lambda_{\geqslant 0} \subseteq \Lambda_{\geqslant 0}^{\prime}$. For that fix $\lambda \in \Lambda_{\geqslant 0}$. Then $\lambda=\sum_{i=1}^{m} k_{i} \omega_{i}$ with some real numbers $k_{i}$. We have to show that $k_{i} \in \mathbb{Z}_{\geqslant 0}$. Now $\lambda \in \Lambda_{\geqslant 0}$ means in particular that $\langle\lambda, \alpha\rangle /\langle\alpha, \alpha\rangle \in \mathbb{Z}_{\leqslant 0}$ for all $\alpha \in \Delta_{k}^{+}$. Hence, $\left\langle\lambda, \alpha_{i}\right\rangle /\left\langle\alpha_{i}, \alpha_{i}\right\rangle \in \mathbb{Z}_{\geqslant 0}$ for all $1 \leqslant i \leqslant n-1$. It remains to show 


\section{S. Gindikin, B. Krötz and G. Ólafsson}

that $\left\langle\lambda, \alpha_{m}\right\rangle /\left\langle\alpha_{m}, \alpha_{m}\right\rangle \in \mathbb{Z}_{\geqslant 0}$. Integrality is clear. Also, since $\mathbb{R}_{\geqslant 0} \cdot \widetilde{\alpha_{m}}$ constitutes a boundary ray of the cone $\Omega$, non-negativity follows.

Define the horospherical Cauchy kernel on $\Xi_{+}$as the function

$$
\mathcal{K}(\xi)=\frac{1}{a_{H}\left(\xi^{-1}\right)^{-\omega_{m}}-1} \cdot \prod_{j=1}^{m-1} \frac{1}{1-a_{H}\left(\xi^{-1}\right)^{\omega_{j}}} \quad\left(\xi \in \Xi_{+}\right) .
$$

In view of Corollary 4.6 and Lemma $5.1(\mathrm{i})$, the function $\mathcal{K}$ is holomorphic, left $H$-invariant and bounded on subsets of the form $G U \cdot \xi_{0}$ for $U \subset A_{+}$compact.

For a function $f \in L^{1}(X)$, this allows us to define its horospherical Cauchy transform by

$$
\widehat{f}(\xi)=\int_{X} f(x) \cdot \mathcal{K}\left(x^{-1} \xi\right) d x \quad\left(\xi \in \Xi_{+}\right) .
$$

We note that the horospherical Cauchy transform is a $G$-equivariant continuous map

$$
L^{1}(X) \rightarrow \mathcal{O}\left(\Xi_{+}\right), \quad f \mapsto \widehat{f} .
$$

Remark 5.2. (a) The horospherical Cauchy kernel $\mathcal{K}$ is tied to the geometry of the minimal cone $\Omega$ : there is no larger $\mathcal{W}_{K}$-invariant open convex cone $\tilde{\Omega}$ such that $\mathcal{K}$ would be holomorphic on $G \exp (\tilde{\Omega}) \cdot \xi_{0}$ (this follows from Lemma 5.1 and $(1.2)$ ). In this context we wish to point the difference to the results of $\S 4$ which are valid for a wider class of convex cones (cf. Remark 4.12).

(b) For each $\lambda \in \Lambda_{0}$ and $\xi \in \Xi_{+}$consider the complex hypersurface

$$
L(\lambda, \xi)=\left\{z \in X_{\mathbb{C}} \mid a_{H}\left(\xi^{-1} z\right)^{\lambda}-1=0\right\}
$$

in $X_{\mathbb{C}}$. Their intersection is $E(\xi)$ and they do not intersect $X$. The singular set of the horospherical Cauchy kernel is the union of the $m$ hypersurfaces $L\left(\omega_{i}, \lambda\right)$ and the edge of this set is just $E(\xi)$. This means that if $f$ is a boundary value of a holomorphic function on $D_{+}$, then $\widehat{f}$ is a residue on $E(\xi)$.

The horospherical Cauchy transform can be decomposed into its constituents associated to the elements $\lambda \in \Lambda_{>0}$. More precisely, for $\lambda \in \Lambda_{>0}$ and $f \in L^{1}(X)$, let us define $\widehat{f}_{\lambda} \in \mathcal{O}\left(\Xi_{+}\right)$by

$$
\widehat{f}_{\lambda}(\xi)=\int_{X} f(x) \cdot a_{H}\left(\xi^{-1} x\right)^{\lambda} d x .
$$

We call the map $\lambda \mapsto \widehat{f}_{\lambda} \in \mathcal{O}\left(\Xi_{+}\right)$the spherical holomorphic Fourier transform of $f$.

LEMma 5.3. The following assertions hold.

(i) Let $U \subset A_{+}$be a compact subset. The series

$$
\sum_{\lambda \in \Lambda_{>0}} a_{H}\left(\xi^{-1}\right)^{\lambda} \quad\left(\xi \in \Xi_{+}\right)
$$

converges uniformly on $G U \cdot \xi_{0} \subset \Xi_{+}$.

(ii) For all $\xi \in \Xi_{+}$, one has

$$
\sum_{\lambda \in \Lambda_{>0}} a_{H}\left(\xi^{-1}\right)^{\lambda}=\mathcal{K}(\xi)
$$


Proof. Uniform convergence on $G U \cdot \xi_{0}$ is immediate from Corollary 4.6 and Lemma 5.1. Summing up the geometric series, one obtains

$$
\begin{aligned}
\sum_{\lambda \in \Lambda_{>0}} a_{H}\left(\xi^{-1}\right)^{\lambda} & =\sum_{k_{1}=\cdots=k_{m-1}=0}^{\infty} \sum_{k_{m}=1}^{\infty} a_{H}\left(\xi^{-1}\right)^{k_{1} \omega_{1}+\cdots+k_{m} \omega_{m}} \\
& =\left(\frac{1}{1-a_{H}\left(\xi^{-1}\right)^{\omega_{m}}}-1\right) \cdot \prod_{j=1}^{m-1} \frac{1}{1-a_{H}\left(\xi^{-1}\right)^{\omega_{j}}} \\
& =\frac{1}{a_{H}\left(\xi^{-1}\right)^{-\omega_{m}}-1} \cdot \prod_{j=1}^{m-1} \frac{1}{1-a_{H}\left(\xi^{-1}\right)^{\omega_{j}}} \\
& =\mathcal{K}(\xi) .
\end{aligned}
$$

We conclude from Lemma 5.3 that the horospherical Cauchy transform of a function $f \in L^{1}(X)$ can be decomposed as

$$
\widehat{f}=\sum_{\lambda \in \Lambda_{>0}} \widehat{f_{\lambda}}
$$

with the right-hand side converging uniformly on compacta.

Remark 5.4. We wish to point out that the horospherical Cauchy kernel is a product of geometrical and not functional analytic reasoning. We emphasize that, in general, not all parameters $\lambda \in \Lambda_{>0}$ in the decomposition of the horospherical Cauchy kernel correspond to unitarizable lowest weight modules (see Remark 5.6 below for a more detailed discussion).

\subsection{Holomorphic Fourier transform on lowest weight representations}

The objective of this section is to give a more detailed discussion of the holomorphic Fourier transform for functions $f \in L^{2}(X)$ that are contained in lowest weight module.

To begin with, we collect some material on spherical unitary lowest weight representations. A reasonable source might be the overview article [KO03].

Let $\left(\pi_{\lambda}, \mathcal{H}_{\lambda}\right)$ be a non-trivial $H$-spherical unitary lowest weight representation of $G$. As before we denote by $v_{\lambda}$ a normalized lowest weight vector. Write $v_{H}$ for the unique $H$-fixed distribution vector that satisfies $\left\langle v_{\lambda}, v_{H}\right\rangle=1$.

We record the fundamental identity

$$
a_{H}(x)^{\lambda}=\left\langle\pi_{\lambda}(x) v_{H}, v_{\lambda}\right\rangle \quad(x \in X),
$$

which allows us to link our geometric discussion in $\S 4$ with representation theory.

Remark 5.5. It follows from Corollary 4.9 that $a_{H}^{\lambda}$ admits a holomorphic extension to the minimal tube $D_{-}$. Traditionally this fact was explained via (5.1) in the context of holomorphic extension of unitary lowest weight modules (see [Nee99]). We wish to point out that Corollary 4.9 asserts more, namely that $\left.a_{H}^{\lambda}\right|_{D_{-}}$is bounded by 1 . In addition, Corollary 4.9 is more geometric, that is, not restricted to unitary parameters $\lambda$.

Pairing the $G$-module of smooth vectors $\mathcal{H}_{\lambda}^{\infty}$ with $v_{H}$ yields the $G$-equivariant embedding

$$
\iota: \mathcal{H}_{\lambda}^{\infty} \rightarrow C^{\infty}(X), \quad v \mapsto\left(x \mapsto\left\langle\pi_{\lambda}\left(x^{-1}\right) v, v_{H}\right\rangle\right)
$$

We say that $\pi_{\lambda}$ is $X$-square integrable if there exists a constant $d_{s}(\lambda)>0$, the spherical formal dimension (cf. [Kro01]), such that $\sqrt{d_{s}(\lambda)} \cdot \iota$ extends to an isometric map $\mathcal{H}_{\lambda} \rightarrow L^{2}(X)$.

$X$-square integrable parameters $\lambda$ are characterized by the condition [OO91]

$$
\langle\lambda-\rho, \alpha\rangle>0 \text { for all } \alpha \in \Delta_{n}^{+} .
$$

Here $\rho=\frac{1}{2} \sum_{\alpha \in \Delta^{+}} m_{\alpha} \alpha$ with $m_{\alpha}=\operatorname{dim}_{\mathbb{C}} \mathfrak{g}_{\mathbb{C}}^{\alpha}$. 


\section{S. Gindikin, B. Krötz And G. Ólafsson}

Likewise we say that $\pi_{\lambda}$ is $X$-integrable if $\iota\left(\mathcal{H}_{\lambda}^{\mathrm{K}-\text { fin }}\right) \subset L^{1}(X)$. Integrability is described by the inequality

$$
\langle\lambda-2 \rho, \alpha\rangle>0 \text { for all } \alpha \in \Delta_{n}^{+} .
$$

The set of parameters $\lambda \in \Lambda_{>0}$ that satisfy condition (5.4) (respectively (5.3)) are denoted by $\Lambda_{1}$ (respectively $\Lambda_{2}$ ). Note that $\Lambda_{1} \subset \Lambda_{2}$.

Remark 5.6. We discuss the lattice $\Lambda_{>0}$ with regard to $\Lambda_{1}$ and $\Lambda_{2}$. One recognizes a strong dependence on the multiplicities $m_{\alpha}$, which we exemplify for three basic cases below. Recall that elements $\lambda \in \Lambda_{>0}$ are described by $\lambda=\sum_{i=1}^{n} \lambda_{i} \omega_{i}$ with $\lambda_{i} \in \mathbb{Z}_{\geqslant 0}$ and $\lambda_{m}>0$. In addition, let us keep in mind that conditions (5.3) and (5.4) are equivalent to $\left\langle\lambda-\rho, \alpha_{m}\right\rangle>0$ and $\left\langle\lambda-2 \rho, \alpha_{m}\right\rangle>0$, respectively.

The equal rank case. In this situation one has $\mathfrak{t}=\mathfrak{c}$ and $m_{\alpha}=1$ for all $\alpha$. Thus, $\rho=\frac{1}{2} \sum_{i=1}^{m} \omega_{i}$ and therefore $\lambda-\rho=\sum_{i=1}^{m}\left(\lambda_{i}-\frac{1}{2}\right) \omega_{i}$. In particular, $\left\langle\lambda-\rho, \alpha_{m}\right\rangle=\left\langle\alpha_{m}, \alpha_{m}\right\rangle\left(\lambda_{m}-\frac{1}{2}\right)$ and thus $\Lambda_{>0} \subset \Lambda_{2}$ as $\lambda_{m} \geqslant 1$ for elements $\lambda \in \Lambda_{>0}$.

The group case. In this situation one has $m_{\alpha}=2$ for all $\alpha$ and so $\rho=\sum_{i=1}^{n} \omega_{i}$. Accordingly we obtain $\left\langle\lambda-\rho, \alpha_{m}\right\rangle=\left\langle\alpha_{m}, \alpha_{m}\right\rangle\left(\lambda_{m}-1\right)$. It follows that $\Lambda_{>0}$ parameterizes the holomorphic discrete series and their limits; in particular, $\Lambda_{2} \subset \Lambda_{>0}$.

The rank one case. Here one has $\Lambda_{>0}=\mathbb{Z}_{>0} \cdot \omega$ and $\rho=\left(m_{\alpha} / 2\right) \alpha$. Thus, $\Lambda_{2}=\left(\mathbb{Z}_{>0}+\left[m_{\alpha} / 2\right]\right) \omega$ and $\Lambda_{2} \subset \Lambda_{>0}$ with equality precisely for $m_{\alpha}=1$, that is, $\mathfrak{g}=\mathfrak{s l}(2, \mathbb{R})$.

For $\lambda \in \Lambda_{2}$, we set $L^{2}(X)_{\lambda}=\iota\left(\mathcal{H}_{\lambda}\right)$.

Lemma 5.7. Let $\lambda, \mu \in \Lambda_{2}$. Fix $v \in \mathcal{H}_{\lambda}$ and define $f(x)=\left\langle\pi_{\lambda}\left(x^{-1}\right) v, v_{H}\right\rangle \in L^{2}(X)_{\lambda}$. Then for all $\xi \in \Xi_{+}$, the function

$$
X \rightarrow \mathbb{C}, \quad x \mapsto f(x) a_{H}\left(\xi^{-1} x\right)^{\mu}
$$

is integrable and

$$
\int_{X} f(x) a_{H}\left(\xi^{-1} x\right)^{\mu} d x=\frac{\delta_{\lambda \mu}}{d_{s}(\lambda)}\left\langle v, \pi_{\lambda}(\bar{\xi}) v_{\lambda}\right\rangle
$$

Here

$$
\pi_{\lambda}(\bar{\xi}) v_{\lambda}=a^{-\lambda} \pi_{\lambda}(g) v_{\lambda} \in \mathcal{H}_{\lambda} \quad \text { for } \xi=g a \cdot \xi_{0}, g \in G \text { and } a \in A_{+} .
$$

Proof. Fix $\xi \in \Xi_{+}$. Holomorphic extension of (5.1) yields

$$
a_{H}\left(\xi^{-1} g\right)^{\mu}=\left\langle\pi_{\lambda}(g) v_{H}, \pi_{\lambda}(\bar{\xi}) v_{\lambda}\right\rangle
$$

for all $g \in G$ and $\xi \in \Xi_{+}$. It follows that $x \mapsto a_{H}\left(\xi^{-1} x\right)^{\mu}$ is square integrable on $X$. Thus, $x \mapsto$ $f(x) a_{H}\left(\xi^{-1} x\right)^{\mu}$ is integrable. Finally, we apply Schur-orthogonality (cf. [Kro01, Proposition 3.2]) and obtain

$$
\begin{aligned}
\int_{X} f(x) a_{H}\left(\xi^{-1} x\right)^{\mu} d x & =\int_{X}\left\langle\pi_{\lambda}\left(x^{-1}\right) v, v_{H}^{\lambda}\right\rangle\left\langle\pi_{\mu}(x) v_{H}^{\mu}, \pi_{\mu}(\bar{\xi}) v_{\mu}\right\rangle d x \\
& =\int_{X}\left\langle\pi_{\lambda}\left(x^{-1}\right) v, v_{H}^{\lambda}\right\rangle \overline{\left\langle\pi_{\mu}\left(x^{-1}\right) \pi_{\mu}(\bar{\xi}) v_{\mu}, v_{H}^{\mu}\right\rangle} d x \\
& =\frac{\delta_{\mu \lambda}}{d_{s}(\lambda)}\left\langle v, \pi_{\lambda}(\bar{\xi}) v_{\lambda}\right\rangle .
\end{aligned}
$$

For $\lambda \in \Lambda_{1}$ let us write $L^{1}(X)_{\lambda}$ for the closure of $\iota\left(\mathcal{H}_{\lambda}^{\mathrm{K}-\mathrm{fin}}\right)$ in $L^{1}(X)$. The following lemma can be understood as an $L^{1}$-version of Schur-orthogonality for the Cauchy transform.

Lemma 5.8. Let $\lambda \in \Lambda_{1}$. Then

$$
\widehat{f}=\widehat{f_{\lambda}} \quad \text { for all } f \in L^{1}(X)_{\lambda} \text {. }
$$


Proof. Fix $f \in L^{1}(X)_{\lambda}$. We have to show that $\widehat{f}_{\mu}=0$ for all $\mu \in \Lambda_{>0} \backslash\{\lambda\}$. For $\mu \in \Lambda_{2}$ this is a consequence of Lemma 5.7. Therefore, we may assume that $\mu \in \Lambda_{>0} \backslash \Lambda_{2}$. This means that condition (5.3) is violated, which we express as

$$
\left\langle\mu-\rho, \alpha_{m}\right\rangle \leqslant 0
$$

We now show that

$$
\widehat{f}_{\mu}(\xi)=\int_{X} f(x) a_{H}\left(\xi^{-1} x\right)^{\mu} d x=0 \quad \text { for all } \xi \in \Xi_{+} .
$$

The above equation has boundary values on $G / M \subset \partial \Xi_{+}$and it will be sufficient to prove that

$$
\widehat{f}_{\mu}(g M)=\int_{X} f(x) a_{H}\left(g^{-1} x\right)^{\mu} d x=0 \quad \text { for all } g \in G .
$$

We compute

$$
\begin{aligned}
\widehat{f}_{\mu}(g M) & =\int_{X} f(x) a_{H}\left(g^{-1} x\right)^{\mu} d x \\
& =\int_{X} f(g x) a_{H}(x)^{\mu} d x \\
& =\int_{T} \int_{X} f(\operatorname{tg} x) a_{H}(t x)^{\mu} d x d t \\
& =\int_{X}\left(\int_{T} t^{\mu} f(\operatorname{tg} x) d t\right) a_{H}(x)^{\mu} d x .
\end{aligned}
$$

To arrive at a contradiction, suppose that $\int_{T} t^{\mu} f(t g x) d t \neq 0$. This can only happen if $\mu$ belongs to the $T$-weight spectrum of $\pi_{\lambda}$. Now the $T$-weights of $\pi_{\lambda}$ are contained in $\lambda+\mathbb{Z}_{\geqslant 0}\left[\Delta^{+}\right]$. Thus, $\mu=\lambda+\gamma$ for some $\gamma \in \Delta^{+}$. However, then

$$
\left\langle\mu-\rho, \alpha_{m}\right\rangle=\left\langle\lambda-\rho, \alpha_{m}\right\rangle+\left\langle\gamma, \alpha_{m}\right\rangle .
$$

Observe that both summands on the right-hand side are positive, the desired contradiction to (5.6).

Remark 5.9 (Analytic continuation). Let $\lambda \in \Lambda_{1}$ and $f \in L^{1}(X)_{\lambda} \cap L^{2}(X)_{\lambda}$. Write $f(x)=$ $\left\langle\pi_{\lambda}\left(x^{-1}\right) v, v_{H}\right\rangle$ for some $v \in \mathcal{H}_{\lambda}$. Then Lemmas 5.7 and 5.8 imply that

$$
\widehat{f}(\xi)=\frac{1}{d_{s}(\lambda)}\left\langle v, \pi_{\lambda}(\bar{\xi}) v_{\lambda}\right\rangle .
$$

Clearly, the right-hand side makes sense for all $v \in \mathcal{H}_{\lambda}$ and all $X$-square integrable parameters $\lambda \in \Lambda_{2}$. We now explain how passing to parameters $\lambda \in \Lambda_{2}$ in (5.7) has a natural explanation in terms of analytic continuation. For this, let $\tilde{G}$ denote the universal cover of $G$. Write $\tilde{\Lambda}_{1}, \tilde{\Lambda}_{2}$ for the sets of $\tilde{G}$-integral parameters that satisfy (5.4), (5.3), respectively. Clearly $\Lambda_{1,2} \subset \tilde{\Lambda}_{1,2}$. The effect of passing to the universal cover is that the parameter spaces involved become continuous in the central variable, that is, there exists constants $0<c_{2}<c_{1}$ such that

$$
\left.\left.\tilde{\Lambda}_{1}\right|_{\mathbb{R} Z_{0}}=\right] c_{1}, \infty\left[\cdot\left(\left.\alpha_{m}\right|_{\mathbb{R} Z_{0}}\right) \quad \text { and }\left.\quad \tilde{\Lambda}_{2}\right|_{\mathbb{R} Z_{0}}=\right] c_{2}, \infty\left[\cdot\left(\left.\alpha_{m}\right|_{\mathbb{R} Z_{0}}\right) .\right.
$$

By the concrete formula for $d_{s}(\lambda)$ from [Kro01, Theorem 4.15], we know that $\lambda \mapsto d_{s}(\lambda)$ is a meromorphic function on $\mathfrak{a}_{\mathbb{C}}^{*}$ that is positive on $\tilde{\Lambda}_{2}$. Now, familiar techniques show that the assignment

$$
\tilde{\Lambda}_{2} \ni \lambda \mapsto \frac{1}{d_{s}(\lambda)}\left\langle v, \pi_{\lambda}(\bar{\xi}) v_{\lambda}\right\rangle \in \mathbb{C}
$$

becomes analytic in the central variable (the Shapovalov form is polynomial in $\lambda$ and it is explained in [Kro99] how to make consistent analytic choices for $v$ and $v_{\lambda}$ in dependence of the central coordinate of $\lambda$ ). 


\section{S. Gindikin, B. Krötz And G. Ólafsson}

Motivated by Remark 5.9, we define the horospherical Cauchy transform for functions $f \in$ $L^{2}(X)_{\lambda}, \lambda \in \Lambda_{2}$ by

$$
\widehat{f}=\widehat{f_{\lambda}}
$$

\subsection{Hyperfunctions and generalized matrix coefficients}

In order to discuss the horospherical Cauchy transform and its inverse in a more comprehensive way, we need some results on the analytic continuation of generalized matrix coefficients of lowest weight representations. Proofs of the facts cited below can be found in [KNO97].

Let $(\pi, \mathcal{H})$ be a unitary lowest weight representation of $G$. Write $\mathcal{H}^{\omega}$ and $\mathcal{H}^{-\omega}$ for the associated $G$-modules of analytic and hyperfunction vectors, respectively. The nature of the $T$-spectrum of $\pi$ shows that $\left.\pi\right|_{T}$ extends holomorphically to $\mathcal{T}_{-}$. Moreover, the so-obtained self-adjoint operators $\pi(a), a \in A_{-}$, are of trace class and strongly mollifying, that is,

$$
\pi(a) \mathcal{H}^{-\omega} \subset \mathcal{H}^{\omega} \quad\left(a \in A_{-}\right) .
$$

Assume that $\pi$ is $H$-spherical and denote by $v_{H}$ the (up to scalar) unique $H$-fixed distribution vector. Let $v \in \mathcal{H}^{-\omega}$ be a hyperfunction vector. We wish to interpret the generalized matrix coefficient $f(x)=\left\langle\pi\left(x^{-1}\right) v, v_{H}\right\rangle$ as a generalized function on $X=G / H$. It follows essentially from (5.8) that the prescription

$$
\tilde{f}\left(g a \cdot x_{0}\right)=\left\langle\pi\left(g^{-1}\right) \pi\left(a^{-1}\right) v, v_{H}\right\rangle \quad \text { for } g \in G \text { and } a \in A_{+}
$$

defines a holomorphic function on $D_{+}=G A_{+} \cdot x_{0}$. The minimal tube $D_{+}$has $X$ as an edge and this allows us to interpret $f$ as the boundary value of $\tilde{f}$. Henceforth, we identify $f$ with the holomorphic function $\tilde{f}$.

Suppose that $\mathcal{H} \subset L^{2}(X)$, that is, $\pi=\pi_{\lambda}$ with $\lambda \in \Lambda_{2}$ and $\mathcal{H}=L^{2}(X)_{\lambda}$. We now show how the horospherical Cauchy transform restricted to $L^{2}(X)_{\lambda}$ can be extended to $L^{2}(X)_{\lambda}^{-\omega} \subset \mathcal{O}\left(D_{+}\right)$. In other words for $\xi \in \Xi_{+}$we wish to give meaning to

$$
\begin{aligned}
\widehat{f}(\xi) & =\int_{X} f(x) a_{H}\left(\xi^{-1} x\right)^{\lambda} d x \\
& =\int_{X}\left\langle\pi\left(x^{-1}\right) v, v_{H}\right\rangle a_{H}\left(\xi^{-1} x\right)^{\lambda} d x
\end{aligned}
$$

as a holomorphic function on $\Xi_{+}$. Express $\xi$ as $\xi=g a \cdot \xi_{0}$ with $g \in G$ and $a \in A_{+}$. By the usual holomorphic change of variables, one obtains that

$$
\begin{aligned}
\widehat{f}(\xi) & =\int_{X} f(x) a_{H}\left(a^{-1} g^{-1} x\right)^{\lambda} d x \\
& =\int_{X}\left\langle\pi\left(x^{-1}\right) \pi\left(g^{-1}\right) \pi\left(a^{-1}\right) v, v_{H}\right\rangle a_{H}(x)^{\lambda} d(x) .
\end{aligned}
$$

Now the last expression is well defined by (5.8). Of course, one has

$$
\widehat{f}(\xi)=\frac{1}{d_{s}(\lambda)}\left\langle\pi\left(a^{-1}\right) \pi\left(g^{-1}\right) v, v_{\lambda}\right\rangle
$$

by the same argument as in Lemma 5.7. Thus, we have shown that the horospherical Cauchy transform on $L^{2}(X)_{\lambda}$ extends to a $G$-equivariant continuous map

$$
L^{2}(X)_{\lambda}^{-\omega} \rightarrow \mathcal{O}\left(\Xi_{+}\right)_{\lambda} .
$$

We conclude this section with a conjecture related to the holomorphic intertwining of $\mathcal{O}\left(D_{+}\right)$ and $\mathcal{O}\left(\Xi_{+}\right)$. It can be seen as a holomorphic analogue of Helgason's conjecture (actually a theorem by $[$ KKMOOT78]). 


\section{HOROSPHERICAL MODEL FOR DISCRETE SERIES}

In order to state the conjecture, some new terminology is needed. Let us call a holomorphic function $f$ on $\Xi_{+}$bounded away from the boundary if its restriction to $g \mathcal{T}_{+} a$ is bounded for all choices of $g \in G$ and $a \in A_{+}$. We denote by $\mathcal{O}_{\text {b.a.b. }}\left(D_{+}\right)$the space of all holomorphic functions on $\Xi_{+}$that are bounded away from the boundary. Note that $\mathcal{O}_{\text {b.a.b. }}\left(D_{+}\right)$is a closed $G$-subspace of the Fréchet space $\mathcal{O}\left(D_{+}\right)$.

Conjecture 1 . Let $\mathbb{D}(X)$ be the algebra of $G$-invariant differential operators on $X$. Naturally, we can view $\mathbb{D}(X)$ as holomorphic differential operators on $X_{\mathbb{C}}$. Write $\mathcal{O}\left(D_{+}\right)_{\lambda}$ for the common holomorphic $\mathbb{D}(X)$-eigenfunctions on $D_{+}$with infinitesimal character $\lambda-\rho$. Let $\lambda \in \Lambda_{2}$. We conjecture that

$$
\mathcal{O}_{\text {b.a.b. }}\left(D_{+}\right)_{\lambda}=L^{2}(X)_{\lambda}^{-\omega} \text {. }
$$

Note that the inclusion ' $\supset$ ' is clear by (5.8).

We have already remarked that $\mathcal{O}\left(\Xi_{+}\right)_{\lambda} \simeq \mathcal{H}_{\lambda}^{-\omega}$ (see [KNO97]). Hence, our conjectured equality means that the horospherical Cauchy transform induces an intertwining isomorphism $\mathcal{O}_{\text {b.a.b. }}\left(D_{+}\right)_{\lambda} \rightarrow$ $\mathcal{O}\left(\Xi_{+}\right)_{\lambda}$.

It is also an interesting problem to formulate (and prove) the conjecture for other parameters.

Remark 5.10. We illustrate Conjecture 1 for the one-sheeted hyperboloid $X=\mathrm{Sl}(2, \mathbb{R}) / \mathrm{SO}(1,1)$. Fix $\lambda \in 2 \mathbb{Z}_{>0}=\Lambda_{2}$ and denote by $\mathcal{H}_{\lambda}$ (respectively $\mathcal{H}_{-\lambda}$ ) the lowest (respectively highest) weight module of $G=\operatorname{Sl}(2, \mathbb{R})$ with lowest (respectively highest) weight $\lambda$ (respectively $-\lambda$ ). Denote by $V_{\lambda-2}$ the finite-dimensional $G$-module of highest weight $\lambda-2$. Write $C^{\infty}(X)_{\lambda}$ for the $\mathbb{D}(X)$-eigenspace with eigenvalue $\lambda-1$. Then,

$$
C^{\infty}(X)_{\lambda} \simeq \mathcal{H}_{\lambda}^{\infty} \oplus \mathcal{H}_{-\lambda}^{\infty} \oplus V_{\lambda-2}
$$

Now, the functions of $\mathcal{H}_{ \pm \lambda}^{\infty}$ extend holomorphically to $D_{ \pm}$but not beyond, whereas the functions of $V_{\lambda-2}$ extend holomorphically to all of $X_{\mathbb{C}}$. One deduces that $\mathcal{O}\left(D_{+}\right)_{\lambda}=\mathcal{H}_{\lambda}^{-\omega} \oplus V_{\lambda-2}$. Finally, the holomorphic functions in $V_{\lambda-2}$ grow exponentially at infinity and hence are not bounded away from the boundary. Thus, $\mathcal{O}_{\text {b.a.b. }}\left(D_{+}\right)_{\lambda}=L^{2}(X)_{\lambda}^{-\omega}$ as conjectured.

\subsection{Inversion of the horospherical Cauchy transform}

To begin with we first have to explain certain facts on incidence geometry between the Shilov boundary $X$ of $D_{+}$and the boundary piece $G / M$ of $\Xi_{+}$.

We keep in mind that we realized $G / M$ in the boundary of $\Xi_{+}$by $G / M \simeq G \cdot \xi_{0} \subset \partial \Xi_{+}$.

Recall the orbits $S(z) \subset \Xi$ from (2.3). For a point $x \in X$ we define the real form of $S(x)$ by

$$
S_{\mathbb{R}}(x)=S(x) \cap G / M .
$$

In view of the incidence relation $(2.4)$, one has

$$
S_{\mathbb{R}}(x)=\{\xi \in G / M \mid \xi \in S(x)\}=\{\xi \in G / M \mid x \in E(\xi)\} .
$$

Lemma 5.11. Let $x=g \cdot x_{0} \in X, g \in G$. Then,

$$
S_{\mathbb{R}}(x)=g H \cdot \xi_{0} \simeq H / M .
$$

Proof. First note that for $x=g \cdot x_{0}$ with $g \in G$ one has $S_{\mathbb{R}}(x)=g \cdot S_{\mathbb{R}}\left(x_{0}\right)$. Hence, it suffices to show that

$$
S_{\mathbb{R}}\left(x_{0}\right)=H \cdot \xi_{0} \simeq H / M
$$

Let $\xi \in S_{\mathbb{R}}\left(x_{0}\right)$ and write $\xi=y \cdot \xi_{0}$ for some $y \in G$. We have to show that $y \in H$ and that $y$ is uniquely determined modulo $M$. First observe that $\xi \in S\left(x_{0}\right)$ means $y N_{\mathbb{C}} \subset H_{\mathbb{C}} N_{\mathbb{C}}$ and so $y \in H_{\mathbb{C}} N_{\mathbb{C}} \cap G$. Now $H_{\mathbb{C}} N_{\mathbb{C}} \cap G=H$ implies $y \in H$. Finally, uniqueness modulo $M$ is immediate from Lemma 2.2. 


\section{S. Gindikin, B. Krötz and G. Ólafsson}

It is possible to view the boundary orbits $S_{\mathbb{R}}(x)$ as certain limits. For $z=g a \cdot x_{0} \in D_{+}$we define

$$
S_{\mathbb{R}}(z)=g a H \cdot \xi_{0} \simeq H / M .
$$

We note that $S_{\mathbb{R}}(z) \subset \Xi_{+}$by (4.10). Furthermore, there is the obvious limit relation

$$
\lim _{A^{+} \ni a \rightarrow \mathbf{1}} S_{\mathbb{R}}(g a \cdot \xi)=S_{\mathbb{R}}\left(g \cdot x_{0}\right) .
$$

Write $d_{z}(\xi)$ for the measure on $S_{\mathbb{R}}(z)$ that is induced from a Haar measure $d(h M)$ on $H / M$ via the identification $S_{\mathbb{R}}(z) \simeq H / M$. Define the space of fiber integrable holomorphic functions on $\Xi_{+}$ by

$$
\mathcal{O}_{\text {f.i. }}\left(\Xi_{+}\right)=\left\{\phi \in \mathcal{O}\left(\Xi_{+}\right)\left|D_{+} \ni z \rightarrow \int_{S_{\mathbb{R}}(z)}\right| \phi(\xi) \mid d_{z}(\xi) \text { is locally bounded }\right\} .
$$

For a function $\phi \in \mathcal{O}_{\text {f.i. }}\left(\Xi_{+}\right)$we define its inverse horospherical transform $\phi^{\vee} \in \mathcal{O}\left(D_{+}\right)$by

$$
\phi^{\vee}(z)=\int_{S_{\mathbb{R}}(z)} \phi(\xi) d_{z}(\xi) \quad\left(z \in D_{+}\right) .
$$

We note that

$$
\mathcal{O}_{\text {f.i. }}\left(\Xi_{+}\right) \rightarrow \mathcal{O}\left(D_{+}\right), \quad \phi \mapsto \phi^{\vee}
$$

is a $G$-equivariant continuous map.

Finally, we define a subset $\Lambda_{c} \subset \Lambda_{2}$ of large parameters by

$$
\Lambda_{c}=\left\{\lambda \in \Lambda_{2} \mid\left(\forall \alpha \in \Delta_{n}^{+}\right)(\lambda-\rho)(\check{\alpha})>2-m_{\alpha}\right\} .
$$

The inversion formula for the horospherical Cauchy transform is based on the following key result.

Lemma 5.12. Let $\lambda \in \Lambda_{c}$. Let $f \in L^{2}(X)_{\lambda}^{-\omega} \subset \mathcal{O}\left(D_{+}\right)$. Then $\widehat{f} \in \mathcal{O}_{\text {f.i }}\left(\Xi_{+}\right)$and

$$
f(z)=d(\lambda) \cdot \int_{S_{\mathbb{R}}(z)} \widehat{f}(\xi) d_{z}(\xi) \quad\left(z \in D_{+}\right) .
$$

In other words, $f=d(\lambda) \cdot(\widehat{f})^{\vee}$.

Proof. Let $f\left(g a \cdot \xi_{0}\right)=\left\langle\pi_{\lambda}\left(a^{-1}\right) \pi_{\lambda}\left(g^{-1}\right) v, v_{H}\right\rangle$ for some $v \in \mathcal{H}_{\lambda}^{-\omega}$. Then by (5.10)

$$
\widehat{f}(\xi)=\frac{1}{d_{s}(\lambda)}\left\langle v, \pi_{\lambda}(\bar{\xi}) v_{\lambda}\right\rangle
$$

As $\lambda \in \Lambda_{c}$, [Kro01, Theorems 2.16 and 3.6] imply that

$$
\int_{H / M} \pi_{\lambda}(h) v_{\lambda} d(h M)=\frac{d_{s}(\lambda)}{d(\lambda)} \cdot v_{H}
$$

with the left-hand side understood as a convergent $\mathcal{H}_{\lambda}^{-\omega}$-valued integral.

Thus, with $z=g a \cdot x_{0}$ one obtains that

$$
\begin{aligned}
\int_{S_{\mathbb{R}}(z)} \widehat{f}(\xi) d_{z}(\xi) & =\int_{H / M} \frac{1}{d_{s}(\lambda)}\left\langle\pi_{\lambda}\left(a^{-1}\right) \pi_{\lambda}\left(g^{-1}\right) v, \pi_{\lambda}(h) v_{\lambda}\right\rangle d(h M) \\
& =\frac{1}{d(\lambda)} \cdot f(z),
\end{aligned}
$$

completing the proof of the lemma. 
Remark 5.13. If $\lambda \in \Lambda_{2} \backslash \Lambda_{c}$ and $0 \neq f \in L^{2}(X)_{\lambda}^{-\omega}$, then the integral $\int_{S_{\mathbb{R}}(z)} \widehat{f}(\xi) d_{z}(\xi)$ does not converge. However, using the results from [Kro01] it can be shown that the identity (5.12) can be analytically continued (cf. Remark 5.9) to all $\lambda \in \Lambda_{2}$. Henceforth, we understand (5.12) as an identity valid for all $\lambda \in \Lambda_{2}$.

The formal dimension $d(\lambda)$ is a polynomial in $\lambda$, explicitly given by [Har56]

$$
d(\lambda)=c \cdot \prod_{\alpha \in \Sigma^{+}}\langle\lambda-\rho(\mathfrak{c}), \alpha\rangle
$$

with $c \in \mathbb{R}$ a constant depending on the normalization of measures.

The right action of $T$ on $\Xi_{+}$induces an identification of $\mathcal{U}\left(\mathfrak{t}_{\mathbb{C}}\right)$ with $G$-invariant differential operators on $\Xi_{+}$. As usual we identify $\mathcal{U}\left(\mathfrak{t}_{\mathbb{C}}\right)$ with polynomial functions on $\mathfrak{t}_{\mathbb{C}}^{*}$. In this way, $d(\lambda)$ corresponds to a $G$-invariant differential operator $\mathcal{L}$ on $\Xi_{+}$that acts along the fibers of $\Xi_{+} \rightarrow F_{+}$ and has constant coefficients in logarithmic coordinates. In particular,

$$
\mathcal{L} \phi=d(\lambda) \cdot \phi \quad\left(\phi \in \mathcal{O}\left(\Xi_{+}\right)_{\lambda}\right) .
$$

Combining this fact with (5.12) we obtain the main result of this paper.

Theorem 5.14. Let $f \in \sum_{\lambda \in \Lambda_{2}} L^{2}(X)_{\lambda}^{-\omega} \subset \mathcal{O}\left(D_{+}\right)$. Then

$$
f=(\mathcal{L} \widehat{f})^{\vee} .
$$

\section{Example of the hyperboloid of one sheet}

This section is devoted to the discussion of the case $G=\mathrm{Sl}(2, \mathbb{R})$ and $H=\mathrm{SO}(1,1)$. Note that $G / H \simeq \mathrm{SO}_{e}(2,1) / \mathrm{SO}_{e}(1,1)$. For what follows, it is inconsequential to assume that $G=\mathrm{SO}_{e}(2,1)$ and $H=\mathrm{SO}_{e}(1,1)$ although the universal complexification of $G=\mathrm{SO}_{e}(2,1)$ is not simply connected.

The map

$$
G / H \rightarrow \mathbb{R}^{3}, \quad g H \mapsto g \cdot\left[\begin{array}{l}
1 \\
0 \\
0
\end{array}\right]
$$

identifies $X=G / H$ with the one-sheeted hyperboloid

$$
X=\left\{x=\left(x_{1}, x_{2}, x_{3}\right)^{\mathrm{T}} \in \mathbb{R}^{3} \mid x_{1}^{2}+x_{2}^{2}-x_{3}^{2}=1\right\} .
$$

The base point $x_{0}$ becomes $(1,0,0)^{\mathrm{T}}$. Let us define a complex bilinear pairing on $\mathbb{C}^{3}$ by

$$
\langle z, w\rangle=z_{1} w_{1}+z_{2} w_{2}-z_{3} w_{3} \quad \text { for } z=\left[\begin{array}{l}
z_{1} \\
z_{2} \\
z_{3}
\end{array}\right], w=\left[\begin{array}{l}
w_{1} \\
w_{2} \\
w_{3}
\end{array}\right] \in \mathbb{C}^{3} .
$$

If we set $\Delta(z)=\langle z, z\rangle$ for $z \in \mathbb{C}^{3}$, then $X=\left\{x \in \mathbb{R}^{3} \mid \Delta(x)=1\right\}$. Further, one has $G_{\mathbb{C}}=$ $\mathrm{SO}(2,1 ; \mathbb{C}) \simeq \mathrm{SO}(3, \mathbb{C})$ and $H_{\mathbb{C}}=\mathrm{SO}(1,1 ; \mathbb{C}) \simeq \mathrm{SO}(2, \mathbb{C})$. Clearly

$$
X_{\mathbb{C}}=G_{\mathbb{C}} / H_{\mathbb{C}}=\left\{z \in \mathbb{C}^{3} \mid \Delta(z)=1\right\} .
$$

Our choice of $T$ will be

$$
T=K=\left\{\left[\begin{array}{ccc}
\cos \theta & \sin \theta & 0 \\
-\sin \theta & \cos \theta & 0 \\
0 & 0 & 1
\end{array}\right] \mid \theta \in \mathbb{R}\right\}
$$




\section{S. Gindikin, B. Krötz and G. Ólafsson}

In particular, $\mathfrak{a}=\mathbb{R} U_{0}$ where

$$
U_{0}=\left[\begin{array}{ccc}
0 & i & 0 \\
-i & 0 & 0 \\
0 & 0 & 0
\end{array}\right]
$$

and $\Delta=\Delta_{n}=\{\alpha,-\alpha\}$ with $\alpha\left(U_{0}\right)=1$. If we demand that $\alpha$ be the positive root, then

$$
N_{\mathbb{C}}=\left\{\left[\begin{array}{ccc}
1-\frac{z^{2}}{2} & i \frac{z^{2}}{2} & i z \\
i \frac{z^{2}}{2} & 1+\frac{z^{2}}{2} & z \\
i z & z & 1
\end{array}\right] \mid z \in \mathbb{C}\right\} .
$$

The homogeneous space $G_{\mathbb{C}} / M_{\mathbb{C}} N_{\mathbb{C}}$ naturally identifies with the isotropic vectors $\Xi=\left\{\zeta \in \mathbb{C}^{3} \backslash\{0\} \mid\right.$ $\Delta(\zeta)=0\}$ via the $G_{\mathbb{C} \text {-equivariant map }}$

$$
G_{\mathbb{C}} / M_{\mathbb{C}} N_{\mathbb{C}} \rightarrow \Xi, \quad g M_{\mathbb{C}} N_{\mathbb{C}} \mapsto g \cdot \zeta_{0} \quad \text { where } \zeta_{0}=\left[\begin{array}{c}
1 \\
-i \\
0
\end{array}\right] .
$$

The correspondence between elements of $\zeta \in \Xi$ and horospheres on $X_{\mathbb{C}}$ is explicitly given by

$$
\zeta \leftrightarrow E(\zeta)=\left\{z \in X_{\mathbb{C}} \mid\langle z, \zeta\rangle=1\right\} .
$$

Elements $\zeta \in \Xi$ can be expressed as $\zeta=\xi+i \eta$ with $\xi, \eta \in \mathbb{R}^{3} \backslash\{0\}$ subject to

$$
\Delta(\xi)=\Delta(\eta) \text { and }\langle\xi, \eta\rangle=0 .
$$

A simple computation yields

$$
\Xi_{+}=\{\zeta=\xi+i \eta \in \Xi \mid \Delta(\xi)=\Delta(\eta)>1\}
$$

and

$$
D_{+}=\left\{z=x+i y \in X_{\mathbb{C}} \mid \Delta(x)>1\right\} .
$$

Next we compute the kernel function.

Lemma 6.1. For all $z \in X_{\mathbb{C}}$ and $\zeta \in \Xi$ one has

$$
a_{H}\left(\zeta^{-1} z\right)^{-\alpha}=\langle z, \zeta\rangle .
$$

Proof. We first show that

$$
a_{H}(g)^{-\alpha}=\left\langle g \cdot x_{0}, \zeta_{0}\right\rangle \quad\left(g \in G_{\mathbb{C}}\right) .
$$

Observe that both sides are holomorphic functions on $G_{\mathbb{C}}$ that are left $N_{\mathbb{C}^{-i n v a r i a n t}}$ and right $H_{\mathbb{C}^{-}}$ invariant. Thus, it is enough to test with elements $a \in A_{\mathbb{C}}$. Then $a_{H}(a)^{-\alpha}=a^{-\alpha}$. On the other hand for

$$
a=\left[\begin{array}{ccc}
\cos \theta & \sin \theta & 0 \\
-\sin \theta & \cos \theta & 0 \\
0 & 0 & 1
\end{array}\right]
$$

with $\theta \in \mathbb{C}$, we specifically obtain

$$
\left\langle a \cdot x_{0}, \zeta_{0}\right\rangle=\left\langle\left[\begin{array}{c}
\cos \theta \\
-\sin \theta \\
0
\end{array}\right],\left[\begin{array}{c}
1 \\
-i \\
0
\end{array}\right]\right\rangle=\cos \theta+i \sin \theta=a^{-\alpha} .
$$

This proves (6.1). 
It is now easy to prove the asserted statement of the lemma. For this, write $\zeta=g \cdot \zeta_{0}$ and $z=y \cdot x_{0}$ for $g, y \in G_{\mathbb{C}}$. Then, (6.1) implies that

$$
a_{H}\left(\zeta^{-1} z\right)^{-\alpha}=a_{H}\left(g^{-1} y\right)^{-\alpha}=\left\langle g^{-1} y \cdot x_{0}, \zeta_{0}\right\rangle=\left\langle y \cdot x_{0}, g \cdot \zeta_{0}\right\rangle=\langle z, \zeta\rangle .
$$

We observe that $\Lambda_{>0}=\Lambda_{2}=\mathbb{Z}_{>0} \cdot \alpha$. Hence, Lemma 6.1 implies that the horospherical Cauchy kernel is

$$
\mathcal{K}(\zeta)=\frac{1}{a_{H}\left(\zeta^{-1}\right)^{-\alpha}-1}=\frac{1}{\left\langle\zeta, x_{0}\right\rangle-1} \quad\left(\zeta \in \Xi_{+}\right)
$$

The horospherical Cauchy transform for $f \in L^{1}(X)$ is given by

$$
\widehat{f}(\zeta)=\int_{X} \frac{f(x)}{\langle\zeta, x\rangle-1} d x \quad\left(\zeta \in \Xi_{+}\right)
$$

with $d x$ the invariant measure on the hyperboloid $X$. Finally, we discuss inversion. Let the inner product on $\mathfrak{a}$ be normalized such that $\langle\alpha, \alpha\rangle=1$ and identify $\mathbb{R}$ with $\mathfrak{a}^{*}$ by means of the bijection $\mathbb{R} \ni \lambda \mapsto \lambda \alpha \in \mathfrak{a}^{*}$. Then, $\Lambda_{>0}=\mathbb{Z}_{>0}$ and $d(\lambda)=\lambda-\frac{1}{2}$. An easy calculation gives

$$
\mathcal{L}=\sum_{j=1}^{3} \zeta_{j} \frac{\partial}{\partial \zeta_{j}}-\frac{1}{2}
$$

For $f \in \sum_{\lambda>0} L^{2}(X)_{\lambda}^{-\omega} \subset \mathcal{O}\left(D_{+}\right)$the inversion formula reads

$$
f(z)=\int_{-\infty}^{\infty}(\mathcal{L} f)\left(\begin{array}{c}
z_{1}-i \frac{z_{2}}{r} \cosh t-i \frac{z_{1} z_{3}}{r} \sinh t \\
z_{2}+i \frac{z_{1}}{r} \cosh t-i \frac{z_{2} z_{3}}{r} \sinh t \\
z_{3}-i r \sinh t
\end{array}\right) d t
$$

where $r=\sqrt{z_{1}^{2}+z_{2}^{2}}$.

\section{ACKnowledgements}

The authors would like to thank the referee for many useful remarks which led to a clarification of the paper. The second author would especially like to thank the people at RIMS, Kyoto for their hospitality during his stay during the autumn of 2004 where part of this work was done. He thanks his host Toshiyuki Kobayashi for giving him the opportunity to do his research in the stimulating atmosphere of RIMS. In addition, it should be mentioned that the material of the paper was lectured on in the RIMS Lie group seminar and enjoyed constructive critique by a knowledgeable audience: a quick look at the facial expressions of T. Kobayashi, K. Nishiyama or E. Opdam was usually enough.

\section{REFERENCES}

Bot57 R. Bott, Homogeneous vector bundles, Ann. of Math. (2) 66 (1957), 203-248.

Cle88 J.-L. Clerc, Fonctions sphériques des espaces symétriques compacts, Trans. Amer. Math. Soc. 306 (1988), 421-431.

EHW83 T. J. Enright, R. Howe and N. Wallach, A classification of unitary highest weight modules in Proceedings of representation theory of reductive groups, Park City, UT, 1982, Progress in Mathematics, vol. 40 (Birkhäuser, Boston, MA, 1983), 97-143.

Gin00

S. Gindikin, Integral Geometry on SL(2; R), Math. Res. Lett. 7 (2000), 417-432.

Gin02 S. Gindikin, An analytic separation of series of representations for $\mathrm{SL}(2 ; \mathbb{R})$, Mosc. Math. J. 2 (2002), 635-645, 804-805. 


\section{HOROSPHERICAL MODEL FOR DISCRETE SERIES}

Gin04 S. Gindikin, Horospherical Cauchy-Radon transform on compact symmetric spaces, Preprint (2004), arXiv:math.RT/0501022, Moscow Math. J., to appear.

Har56 Harish-Chandra, Representations of semisimple Lie groups. VI. Integrable and squareintegrable representations, Amer. J. Math. 78 (1956), 564-628.

Hel84 S. Helgason, Groups and geometric analysis (Academic Press, Reading, MA, 1984).

Hel94 S. Helgason, Geometric analysis on symmetric spaces, Mathematical Surveys and Monographs, vol. 39 (American Mathematical Society, Providence, RI, 1994).

HO96 J. Hilgert and G. Ólafsson, Causal symmetric spaces, geometry and harmonic analysis (Academic Press, Reading, MA, 1996).

HOO91 J. Hilgert, G. Ólafsson and B. Ørsted, Hardy spaces on affine symmetric spaces, J. reine angew. Math. 415 (1991), 189-218.

Hoc65 G. Hochschild, The structure of Lie groups (Holden-Day, San Francisco, CA, 1965).

KKMOOT78 M. Kashiwara, A. Kowata, K. Minemura, K. Okamoto, T. Oshima and M. Tanaka, Eigenfunctions of invariant differential operators on a symmetric space, Ann. of Math. (2) 107 (1978), 1-39.

Kro99 B. Krötz, Norm estimates for unitarizable highest weight modules, Ann. Inst. Fourier (Grenoble) 49 (1999), 1241-1264.

Kro01 B. Krötz, Formal dimension for semisimple symmetric spaces, Compositio Math. 125 (2001), $155-191$.

KNO97 B. Krötz, K.-H. Neeb and G. Ólafsson, Spherical representations and mixed symmetric spaces, Represent. Theory 1 (1997), 424-461.

KO03 B. Krötz and G. Ólafsson, The c-function for non-compactly causal symmetric spaces and its relations to harmonic analysis and representation theory in Lie groups and symmetric spaces, American Mathematical Society Translations Series 2, vol. 210 (American Mathematical Society, Providence, RI, 2003), 171-194.

Nee94 K.-H. Neeb, A convexity theorem for semisimple symmetric spaces, Pacific J. Math. 162 (1994), 305-349.

Nee99 K.-H. Neeb, On the complex geometry of invariant domains in complexified symmetric spaces, Ann. Inst. Fourier (Grenoble) 49 (1999), 177-225.

O088 G. Ólafsson and B. Ørsted, The holomorphic discrete series for affine symmetric spaces, J. Funct. Anal. 81 (1988), 126-159.

OO91 G. Ólafsson and B. Ørsted, The holomorphic discrete series of an affine symmetric space and representations with reproducing kernels, Trans. Amer. Math. Soc. 326 (1991), 385-405.

Osh89 T. Oshima, Asymptotic behavior of Flensted-Jensen's spherical trace functions with respect to spectral parameters, in Algebraic analysis, geometry, and number theory, Baltimore, MD, 1988 (Johns Hopkins University Press, Baltimore, MD, 1989), 313-323.

Simon Gindikin gindikin@math.rutgers.edu

Department of Mathematics, Rutgers University, New Brunswick, NJ 08903, USA

Bernhard Krötz kroetz@mpim.mpg-bonn.de

Max-Planck-Institut für Mathematik, Vivatsgasse 7, D-53111 Bonn, Germany

Gestur Ólafsson olafsson@math.Isu.edu

Department of Mathematics, Louisiana State University, Baton Rouge, LA 70803, USA 